LAWRENCE LIVERMORE N A T IO N A L LABORATORY
A High Order Mixed Vector Finite Element Method for Solving the Time Dependent Maxwell Equations on Unstructured Grids

Robert N. Rieben, Garry H. Rodrigue, Daniel A. White

March 17, 2004

Journal of Computational Physics 
This document was prepared as an account of work sponsored by an agency of the United States Government. Neither the United States Government nor the University of California nor any of their employees, makes any warranty, express or implied, or assumes any legal liability or responsibility for the accuracy, completeness, or usefulness of any information, apparatus, product, or process disclosed, or represents that its use would not infringe privately owned rights. Reference herein to any specific commercial product, process, or service by trade name, trademark, manufacturer, or otherwise, does not necessarily constitute or imply its endorsement, recommendation, or favoring by the United States Government or the University of California. The views and opinions of authors expressed herein do not necessarily state or reflect those of the United States Government or the University of California, and shall not be used for advertising or product endorsement purposes. 


\title{
A High Order Mixed Vector Finite Element Method for Solving the Time Dependent Maxwell Equations on Unstructured Grids
}

\author{
R. N. Rieben ${ }^{a, b, *}$, G. H. Rodrigue ${ }^{a, b}$, D. A. White ${ }^{c}$ \\ ${ }^{a}$ University of California at Davis, Department of Applied Science \\ ${ }^{\mathrm{b}}$ Institute for Scientific Computing Research, Lawrence Livermore National Laboratory \\ ${ }^{\mathrm{c}}$ Defense Sciences Engineering Division, Lawrence Livermore National Laboratory
}

\begin{abstract}
We present a mixed vector finite element method for solving the time dependent coupled Ampere and Faraday laws of Maxwell's equations on unstructured hexahedral grids that employs high order discretization in both space and time. The method is of arbitrary order accuracy in space and up to 5th order accurate in time, making it well suited for electrically large problems where grid anisotropy and numerical dispersion have plagued other methods. In addition, the method correctly models both the jump discontinuities and the divergence-free properties of the electric and magnetic fields, is charge and energy conserving, conditionally stable, and free of spurious modes. Several computational experiments are performed to demonstrate the accuracy, efficiency and benefits of the method.
\end{abstract}

Key words:

Computational electromagnetics, Maxwell's equations, vector finite elements, high order methods, $H(\mathrm{Curl})$ and $H($ Div $)$ - conforming methods, discrete differential forms, spurious modes, numerical dispersion

* Corresponding author.

Email address: rieben1@llnl.gov (R. N. Rieben).

1 This work was performed under the auspices of the U.S. Department of Energy by the University of California, Lawrence Livermore National Laboratory under contract No. W7405-Eng-48, and under the U.S. Air Force Contract No. F49620-01-1-0327 


\section{Introduction}

Methods for solving the full vector form of the coupled first order Maxwell equations (or the Ampere and Faraday laws) have been in existence since the original Yee scheme [1]. In the standard formulation, the electric field is discretized over a point grid that is offset both spatially and temporally from a "dual-grid" over which the magnetic field is discretized. The curl operator is approximated with a second order central difference formula. The system is integrated in time via a second order "leap frog" update method where field values at a current time step are calculated in terms of the field values at previous time steps [1]. The method is conditionally stable and consistent, leading to second order convergence as the grid is refined in space and time [2].

In addition, the Yee scheme has several desirable properties from a computational physics viewpoint, namely: conservation of numerical charge and energy, proper modeling of field discontinuities, divergence free fields and no spurious modes. The dual-grid formulation facilitates conservation of numerical charge; at every interior primary node, the sum of the electric field components on the edges connected to that node is zero, hence there is no net charge in the mesh interior. Likewise, at every dual node, the sum of the magnetic field components on the dual-edges connected to that node is zero. Since the dual-edges are the faces of the primary grid, this implies a net zero flux through the primary faces and hence divergence free magnetic fields. Use of the second order accurate leap-frog method leads to conservation of numerical energy in a time averaged sense. To account for open-region problems (e.g. the Sommerfeld radiation boundary condition), several techniques have been developed such as the absorbing boundary condition (ABC) and the perfectly matched layer (PML) [3], [4]. The combination of these properties along with the method's efficiency, elegance and ease of implementation have established the FDTD as the benchmark method in computational electromagnetics (CEM) to which all other methods are compared.

Despite these benefits, the method is certainly not without it limitations, and it is precisely these limitations which have lead to the development of new, more advanced methods. The shortcomings of the FDTD method are mainly twofold. The first is the restriction of the method to Cartesian grids (or those which can be mapped to Cartesian grids). Objects with curved boundaries must be gridded in a "stair-step" manner and it has been shown that such approximations can give very poor results [5], [6]. In order to circumvent this limitation, there have been several attempts to generalize the FDTD method to unstructured grids, including the modified finite volume (MFV) technique [7], [8] and the discrete surface integral (DSI) method [9]. However, a serious defect of these methods is the existence of late time growth of the solution amplitude (often called a "late-time instability") for problems on non-orthogonal grids, irrespective of the time step used. This instability is caused by the non-symmetric discretization of the curl-curl operator [10]. The 
use of dissipative time integration schemes (e.g. artificial viscosity or filtering) can dampen the growth of this non-physical solution, but this results in a violation of numerical charge and energy conservation.

The second limitation of the FDTD method is numerical dispersion. Numerical dispersion is the nonphysical dependence of computed wave propagation velocity on frequency; resulting in a cumulative growth in global phase error for timedependent problems (also called the pollution effect [11]). The consequences of this purely numerical phenomenon are present in any grid based method; the goal therefore is to reduce its effect as much as possible. This is typically accomplished by adding grid points to a mesh to more fully resolve the spatial and temporal nature of an electromagnetic wave. However, for certain "electrically large" problems in which several wavelengths span the computational domain or for certain broadband applications, it can become prohibitively expensive to achieve a prescribed tolerance for numerical dispersion error using standard grid refinement. A more efficient way to reduce numerical dispersion is to employ a higher order method. High-order spatial discretizations can yield extremely accurate and efficient results for certain problems with smoothly curved boundaries, and they can drastically reduce the effects of numerical dispersion [12], [13], [14], [15]. Extensions of the FDTD method to higher order versions have been published, such as the fourth order accurate methods of [16], [17], [18] and [19], however these methods were developed for 2D orthogonal grids and often have difficulties maintaining accuracy at material interfaces and PEC boundaries due to the high order finite difference stencils. Recent advances in the finite element method have lead to more generalized high order implementations on unstructured grids, while eliminating the presence of spurious-modes which are encountered whenever scalar (or nodal) basis functions are improperly used to discretize the curl-curl operator [20], [21]. These include the spectral penalty methods of [22] and the high order interpolatory vector basis functions of [23].

In this paper, we propose a high order mixed vector finite element method for solving Maxwell's equations directly in the time domain. The method has several key benefits. High order spatial discretization is achieved by employing the high order interpolatory basis functions of [24], derived from the Nédeléc polynomial spaces [25]. These vector basis functions satisfy the properties of the recently proposed differential forms based approach for constructing 1-form (also known as curl-conforming, $\mathrm{H}(\mathrm{Curl})$ or "edge") bases and 2-form (also known as divergenceconforming, $H($ Div $)$ or "face") bases [26]. For the Galerkin procedure applied to either the frequency domain or time dependent Maxwell equations, there are significant advantages to both 1-form and 2-form finite element basis functions [27]; including the proper modeling of the jump discontinuity of field intensities and flux densities across material interfaces, the elimination of spurious modes in eigenvalue computations and the conservation of charge in time-dependent simulations [27]. High order temporal discretization is achieved via the symplectic integration methods of [28]. Symplectic methods have the benefit of conserving total electro- 
magnetic field energy and are therefore preferred over dissipative methods (such as Runge-Kutta) in applications that require high-accuracy and energy conservation over long periods of time integration.

The structure of the paper is as follows. In Section 2 we introduce the precise form of the time dependent PDEs we are concerned with. In Section 3 we present the variational formulation of the PDE and apply Galerkin's method to yield a linear system of ODEs. In Section 4 we present the key ingredients necessary to discretize the problem in space, including the finite element basis functions, degrees of freedom, bilinear forms and global assembly process. In Section 5 we explain the symplectic time integration process used to numerically integrate the time dependent algebraic equations which are produced from the spatial discretization process. We also discuss the nature of stability and numerical energy and charge conservation of the method. In Section 6 we provide several computational examples ranging from simple resonant cavity simulations to more complicated wave guide simulations which demonstrate the properties of the proposed method. Finally, in Section 7 we conclude the paper.

\section{The Coupled Ampere-Faraday Equations}

We begin with the time dependent Ampere-Faraday equations which describe the spatial and temporal evolution of the 1-form electric field intensity $\mathbf{E}$ and the 2-form magnetic flux density $\mathbf{B}$, given by

$$
\begin{array}{rlrl}
\varepsilon \frac{\partial}{\partial t} \mathbf{E} & =\nabla \times\left(\mu^{-1} \mathbf{B}\right)-\sigma \mathbf{E}-\mathbf{J} \text { in } \Omega \\
\frac{\partial}{\partial t} \mathbf{B} & =-\nabla \times \mathbf{E} & \text { in } \Omega
\end{array}
$$

We impose the physical restriction that there are no free electric or magnetic charges in the problem domain; and therefore, require only the presence of current (or voltage) sources, yielding the constraints

$$
\begin{array}{r}
\nabla \cdot(\varepsilon \mathbf{E})=0 \text { in } \Omega \\
\nabla \cdot \mathbf{B}=0 \text { in } \Omega
\end{array}
$$


In order to fully define the problem, the field variables are subject to the following initial-boundary values

$$
\begin{aligned}
\hat{\mathbf{n}} \times \mathbf{E} & =\mathbf{E}_{b c} \text { on } \partial \Omega \\
\mathbf{E}(t) & =\mathbf{E}_{i c} \text { at } t=t_{0} \\
\mathbf{B}(t) & =\mathbf{B}_{i c} \text { at } t=t_{0}
\end{aligned}
$$

The symbol $\Omega$ is a three dimensional domain in which the fields exist, $\partial \Omega$ is the two dimensional boundary of the domain, $\hat{\mathbf{n}}$ is the outwardly directed unit normal of this boundary and $\mathbf{J}$ is a time and space dependent 2 -form current flux density. The symbols $\varepsilon, \mu$ and $\sigma$ are the dielectric function, magnetic permeability and electrical conductivity respectively; describing the material properties of the medium in which the fields exist. These parameters are free to be tensor valued functions of space; however, we impose the restriction that they are linear and independent of time. The value $\mathbf{E}_{b c}$ represents an arbitrary boundary condition imposed on the electric field intensity and can range anywhere from the simple case of a perfect electric conductor (PEC) to more complicated voltage sources or absorbing boundary conditions (ABC). The values $\mathbf{E}_{i c}$ and $\mathbf{B}_{i c}$ represent the initial conditions of the problem, and $t_{0}$ is the initial time. Note that if the current source(s) described by $\mathbf{J}$ are divergence free (implying conservation of charge), then the divergence constraints of (2) will be satisfied for all time, provided that the initial condition data is divergence free.

\section{Formulation of The Method}

\subsection{Variational Formulation}

We now apply a variational formulation to the coupled first order field equations of (1). We begin by computing the inner product of each term in the electric field intensity equation with a 1 -form test function $\mathbf{E}^{\prime}$, and each term in the magnetic flux density equation with a 2 -form test function $\mathbf{B}^{\prime}$. The resulting equations are then integrated over a volume to yield

$$
\begin{aligned}
& \int_{\Omega} \varepsilon \frac{\partial}{\partial t} \mathbf{E} \cdot \mathbf{E}^{\prime}=\int_{\Omega} \nabla \times\left(\frac{1}{\mu} \mathbf{B}\right) \cdot \mathbf{E}^{\prime}-\int_{\Omega} \sigma \mathbf{E} \cdot \mathbf{E}^{\prime}-\int_{\Omega} \mathbf{J} \cdot \mathbf{E}^{\prime} \\
& \int_{\Omega} \frac{1}{\mu} \frac{\partial}{\partial t} \mathbf{B} \cdot \mathbf{B}^{\prime}=-\int_{\Omega} \frac{1}{\mu} \nabla \times \mathbf{E} \cdot \mathbf{B}^{\prime}
\end{aligned}
$$


Using integration by parts and the Stokes theorem yields the following linear functionals

$$
\begin{aligned}
& \int_{\Omega} \varepsilon \frac{\partial}{\partial t} \mathbf{E} \cdot \mathbf{E}^{\prime}=\int_{\Omega} \frac{1}{\mu} \mathbf{B} \cdot \nabla \times \mathbf{E}^{\prime}-\int_{\Omega} \sigma \mathbf{E} \cdot \mathbf{E}^{\prime}-\int_{\Omega} \mathbf{J} \cdot \mathbf{E}^{\prime}-\oint_{\partial \Omega}\left(\frac{1}{\mu} \mathbf{B} \times \mathbf{E}^{\prime}\right) \cdot \hat{\mathbf{n}} \\
& \int_{\Omega} \frac{1}{\mu} \frac{\partial}{\partial t} \mathbf{B} \cdot \mathbf{B}^{\prime}=-\int_{\Omega} \frac{1}{\mu} \nabla \times \mathbf{E} \cdot \mathbf{B}^{\prime}
\end{aligned}
$$

These linear functionals are appropriate for use in a Galerkin finite element procedure.

Note that by using integration by parts and the Stokes theorem, it has been assumed that the field variables and their respective test functions have a certain amount of smoothness, namely that

$$
\begin{aligned}
& \mathbf{E} \in\left\{H(\text { Curl }): \int_{\Omega} \varepsilon \mathbf{E} \cdot \mathbf{E}+\int_{\Omega}(\varepsilon \nabla \times \mathbf{E}) \cdot(\nabla \times \mathbf{E})<\infty\right\} \\
& \mathbf{B} \in\left\{H(\text { Div }): \int_{\Omega} \frac{1}{\mu} \mathbf{B} \cdot \mathbf{B}+\int_{\Omega}\left(\frac{1}{\mu} \nabla \cdot \mathbf{B}\right) \cdot(\nabla \cdot \mathbf{B})<\infty\right\}
\end{aligned}
$$

The constraints of (6) and (7) have a very relevant physical interpretation for the case of Maxwell's equations. The first term in the constraints above states that the electric and magnetic field energies must remain finite, while the second term in the constraints is a direct consequence of Ampere's law and Faraday's law, implying that the time varying 2-form electric and magnetic flux densities must be finite as well. There are further implications from the constraints of (6) and (7). Consider the situation of a material interface with a surface separating the two materials with differing dielectric constants. In the absence of a surface charge density, Gauss' law implies that the normal component of the electric flux density $\varepsilon \mathbf{E}$ is continuous, therefore the normal component of electric field intensity $\mathbf{E}$ is discontinuous. Conversely, Faraday's Law implies that the tangential component of the electric field intensity $\mathbf{E}$ is continuous, therefore the tangential component of the electric flux density $\mathbf{\varepsilon} \mathbf{E}$ is discontinuous.

\subsection{Spatially Discretized PDE}

In this paper, we use polynomial basis functions of arbitrary degree to discretize the function spaces $H(\mathrm{Curl})$ and $H(\mathrm{Div})$. Let $\Sigma_{h}$ be a piecewise discretization of the physical domain $\Omega$ of (1) using a mesh of hexahedral elements of characteristic volume $\Delta h$. The field variables are then approximated over each element $\Sigma \in \Sigma_{h}$ by basis function expansions of the form 


$$
\begin{aligned}
& \mathbf{E}(\mathbf{r}, t) \approx \sum_{i} e_{i}(t) \mathbf{w}_{i}(\mathbf{r}), \quad \mathbf{w}_{i} \in W_{h} \subset H(C u r l) \\
& \mathbf{B}(\mathbf{r}, t) \approx \sum_{i} b_{i}(t) \mathbf{f}_{i}(\mathbf{r}), \quad \mathbf{f}_{i} \in F_{h} \subset H(\text { Div }) \\
& \quad \text { for } \quad \mathbf{r} \in \Sigma, \quad t_{0} \leq t \leq t_{\text {fin }}
\end{aligned}
$$

where $e_{i}(t)$ are the time dependent 1-form degrees of freedom, $b_{i}(t)$ are the time dependent 2-form degrees of freedom, $\mathbf{w}_{i}(\mathbf{r})$ are the spatially dependent 1-form polynomial basis functions and $\mathbf{f}_{i}(\mathbf{r})$ are the spatially dependent 2-form polynomial basis functions.

Applying Galerkin's method to the variational formulations of (4) and (5) yields the following linear system of first order ordinary differential equations (ODEs)

$$
\begin{aligned}
M_{\varepsilon} \frac{\partial}{\partial t} e & =K^{T} M_{\mu} b-M_{\sigma} e-M_{\varepsilon} j \\
\frac{\partial}{\partial t} b & =-K e
\end{aligned}
$$

where $e$ and $b$ represent the discrete differential 1-form and 2-form electric and magnetic fields respectively, $K$ is a rectangular matrix representing the discrete curl operator, $M_{\varepsilon}$ is a symmetric positive definite (SPD) 1-form mass matrix computed using the material property function $\varepsilon$ to represent the dielectric properties, $M_{\sigma}$ is the SPD 1-form mass matrix computed using the material property function $\sigma$ to represent the electric conductivity, $M_{\mu}$ is the SPD 2-form mass matrix computed using the material property function $\mu$ to represent the magnetic permeability and $j$ is the discrete 2 -form time dependent current source. In the next section, we will present the key components necessary for constructing these matrices and vectors on unstructured hexahedral meshes.

\section{High Order Spatial Discretization}

Here we present the key components for assembling the finite element matrices of (10) for arbitrary order accuracy. We use the symbol $p$ to denote the polynomial degree of a basis function. For a polynomial basis of degree $p$, the discrete PDE of (10) will be $p+1$ order accurate in space. We follow the work of Ciarlet [29] and adhere to the strict mathematical definition of a finite element as a set of three distinct objects $(\Sigma, \mathcal{P}, \mathcal{A})$ such that:

- $\Sigma$ is the polyhedral domain over which the element is defined

- $\mathcal{P}$ is a finite dimensional polynomial space from which basis functions are constructed

- $\mathcal{A}$ is a set of linear functionals (Degrees of Freedom) dual to $\mathcal{P}$ 
Separating the element $\Sigma$ from the basis allows for curvilinear elements of arbitrary geometry order. Precise definitions for $\mathcal{A}$ are needed to define projection and interpolation operators. These are necessary for applying boundary conditions and performing normed error analysis. To construct the local (or single element) matrices, we present explicit bilinear forms. To assemble the local matrix results into a global linear system in a conforming manner, we also present a global assembly process.

\subsection{Polynomials}

We use interpolatory polynomials as the building blocks for the high order vector basis functions used in the formulation of (10). It is also possible to use hierarchical basis functions instead, such as those of [30] and [31]. The Lagrange interpolatory polynomial of degree $p$ is defined by a distinct set of $p+1$ real valued interpolation points denoted by the symbol $X$, such that $X=\left\{X_{0}, X_{1}, \ldots, X_{p}\right\}$. The polynomial is constructed in such a way that it has a value of unity at interpolation point $i$ and a value of zero at every other interpolation point. The precise definition for the Lagrange interpolatory polynomial of degree $p$ is given by

$$
L_{i}^{p}(x ; X)=\prod_{\substack{j=0 \\ j \neq i}}^{p} \frac{\left(x-X_{j}\right)}{\left(X_{i}-X_{j}\right)}
$$

The set of $p+1$ interpolation points, $X$, can at this point be arbitrary; however as show in [24], the use of special non-uniform points based on the zeros of Chebyshev polynomials yield substantially improved conditioning of finite element matrices in contrast to standard basis functions which use uniformly distributed interpolation points.

\section{2 $\Sigma$ - Element Topology and Geometry}

All hexahedral elements (including curved elements) in a physical mesh are topologically equivalent to a reference hexahedral element. In order to make integration over the reference element as simple as possible, we adopt a standard Cartesian coordinate system with an origin at the point $(0,0,0)$ as our reference coordinate system. Throughout the remainder of this paper, all objects explicitly defined with respect to this reference coordinate system will be accented with a hat symbol. Let $\hat{\Sigma}$ denote the unit hexahedron such that

$$
\hat{\Sigma}=\left\{\left(\hat{r}_{1}, \hat{r}_{2}, \hat{r}_{3}\right) ; 0 \leq\left(\hat{r}_{1}, \hat{r}_{2}, \hat{r}_{3}\right) \leq 1\right\}
$$


There exists a mapping $\Phi$ from the reference element $\hat{\Sigma}$ to an actual mesh element $\Sigma$. This mapping (defined by interpolatory shape functions) and its Jacobian are defined as

$$
\mathbf{r}=\Phi(\hat{\mathbf{r}}) ; \quad J_{i, j}=\frac{\partial r_{j}}{\partial \hat{r}_{i}}
$$

where $\hat{\mathbf{r}} \in \hat{\Sigma}$ and $\mathbf{r} \in \Sigma$. For hexahedral elements, these shape functions can be constructed in a very efficient manner using a tensor product of the Lagrange interpolatory polynomials. A mapping of order $s$ can be constructed as

$$
\begin{aligned}
\Phi(\hat{\mathbf{r}}) & =\sum_{i=1}^{(s+1)^{3}} n_{i} N_{i}(\hat{\mathbf{r}}) \\
\{N(\hat{\mathbf{r}})\} & =\left\{L_{i}^{s}\left(\hat{r}_{1}\right) L_{j}^{s}\left(\hat{r}_{2}\right) L_{k}^{s}\left(\hat{r}_{3}\right) ; i, j, k=0, \ldots, s\right\}
\end{aligned}
$$

where $n_{i}$ are the global coordinates of the $(s+1)^{3}$ vertices used to define the global hexahedron and the set of interpolation points $X$ (omitted for clarity) are uniformly distributed. The geometry order $s$ is independent of the basis order and it determines the degree of distortion of the global mesh element. For example a mapping of order $s=1$ implies a coordinate transformation to a linearly distorted element defined by the global coordinates of 8 vertices, while a mapping of order $s=2$ implies a transformation to a quadratically distorted element defined by the global coordinates of 27 vertices.

\section{$4.3 \mathcal{P}$ - Polynomial Spaces and Basis Functions}

In this section we present explicit formulae for the construction of high order interpolatory 1-form and 2-forms basis functions (or discrete differential forms). These basis functions are derived from the curl and divergence conforming polynomial spaces originally proposed by Nédeléc [25].

For clarity, we will denote the three independent variables in the reference system using the standard Cartesian notation of $(\hat{x}, \hat{y}, \hat{z})$. In addition, we will denote contravariant basis vectors as $\hat{\mathbf{x}}, \hat{\mathbf{y}}$ and $\hat{\mathbf{z}}$, while covariant basis vectors will be denoted as $\hat{\mathbf{X}}, \hat{\mathbf{Y}}$ and $\hat{\mathbf{Z}}$. This is a trivial distinction since these basis vectors are identical, have unit magnitude, and are constant over the domain of the reference hexahedron. However, we make the distinction to emphasize the different transformation properties of the bases associated with these vectors. We also omit the interpolation points $X$ from the Lagrange interpolatory polynomials, implying that the set may be arbitrary; however, for improved matrix conditioning the extended Chebyshev points of [24] should be used. In order to ensure the proper conformity across element to element interfaces, it is crucial that the basis functions (and consequently 
the degrees of freedom) be associated with the various sub-simplices of the element (e.g. nodes, edges, faces, etc ...). This property is referred to as locality [26]. As such, we decompose all of the bases into sub-sets corresponding to the sub-simplex they are associated with. The dimensions of these subsets will in general be number of sub-simplices per element times number of degrees of freedom per sub-simplex.

\subsubsection{1-form Basis Functions}

Let $\hat{W}_{h}$ denote a 1 -form basis on the reference element, with individual basis functions denoted as $\hat{\mathbf{w}}_{i}$ such that $\hat{\mathbf{w}}_{i} \in \hat{W}_{h}$. In order to satisfy the locality property, we can break this set of basis functions into three mutually disjoint subsets such that

$$
\hat{W}_{h}=\hat{W}_{e} \cup \hat{W}_{f} \cup \hat{W}_{v}
$$

where the subscripts $e, f$ and $v$ denote the edges, faces and volume of the reference element respectively. For 1-forms, locality implies that the edge basis functions should have non-vanishing tangential components along one and only one edge. The face basis functions will have non-vanishing tangential components along one and only one face with no tangential components along any edges. Finally, the volume basis functions will have no tangential components along either edges or faces. The 1-form edge basis functions of polynomial degree $p$ are given by

$$
\hat{W}_{e}=\left\{\begin{array}{l}
L_{i}^{p}(\hat{y}) L_{j}^{p}(\hat{z}) L_{k}^{p-1}(\hat{x}) \hat{\mathbf{x}} \\
L_{i}^{p}(\hat{x}) L_{j}^{p}(\hat{z}) L_{k}^{p-1}(\hat{y}) \hat{\mathbf{y}} \quad i, j=0, p ; k=0, \ldots, p-1 \\
L_{i}^{p}(\hat{x}) L_{j}^{p}(\hat{y}) L_{k}^{p-1}(\hat{z}) \hat{\mathbf{z}}
\end{array}\right.
$$

This set of functions is grouped into three sub-sets, one for each contravariant basis vector. The indices $i$ and $j$ loop over the 4 edges that are tangent to these basis vectors. The index $k$ loops over the $p$ basis functions per edge for a total of $12 p$. The 1 -form face basis functions of polynomial degree $p$ are given by

$$
\begin{aligned}
& \hat{W}_{f}=\left\{\begin{array}{l}
L_{i}^{p}(\hat{x}) L_{j}^{p}(\hat{z}) L_{k}^{p-1}(\hat{y}) \hat{\mathbf{y}} \\
L_{i}^{p}(\hat{x}) L_{j}^{p}(\hat{y}) L_{k}^{p-1}(\hat{z}) \hat{\mathbf{z}} \\
L_{i}^{p}(\hat{y}) L_{j}^{p}(\hat{z}) L_{k}^{p-1}(\hat{x}) \hat{\mathbf{x}} \\
L_{i}^{p}(\hat{y}) L_{j}^{p}(\hat{x}) L_{k}^{p-1}(\hat{z}) \hat{\mathbf{z}} \\
L_{i}^{p}(\hat{z}) L_{j}^{p}(\hat{y}) L_{k}^{p-1}(\hat{x}) \hat{\mathbf{x}} \\
L_{i}^{p}(\hat{z}) L_{j}^{p}(\hat{x}) L_{k}^{p-1}(\hat{y}) \hat{\mathbf{y}}
\end{array}\right. \\
& i=0, p ; j=1, \ldots, p-1 ; k=0, \ldots, p-1
\end{aligned}
$$


This set of functions is grouped into six sub-sets, two for each face representing the contravariant basis vectors that are in the plane of that face. The index $i$ loops over the 2 faces that are coplanar to these basis vectors. The indices $j$ and $k$ loop over the $2 p(p-1)$ basis functions per face for a total of $12 p(p-1)$. Finally, there will be a total of $3 p(p-1)^{2}$ interpolatory basis functions that are internal to the reference element given by

$$
\hat{W}_{v}=\left\{\begin{array}{l}
L_{i}^{p}(\hat{y}) L_{j}^{p}(\hat{z}) L_{k}^{p-1}(\hat{x}) \hat{\mathbf{x}} \\
L_{i}^{p}(\hat{x}) L_{j}^{p}(\hat{z}) L_{k}^{p-1}(\hat{y}) \hat{\mathbf{y}} \quad i, j=1, \ldots, p-1 ; k=0, \ldots, p-1 \\
L_{i}^{p}(\hat{x}) L_{j}^{p}(\hat{y}) L_{k}^{p-1}(\hat{z}) \hat{\mathbf{z}}
\end{array}\right.
$$

\subsubsection{2-form Basis Functions}

Let $\hat{F}_{h}$ denote a 2 -form basis on the reference element, with individual basis functions denoted as $\hat{\mathbf{f}}_{i}$ such that $\hat{\mathbf{f}}_{i} \in \hat{F}_{h}$. In order to satisfy the locality property, we can break this set of basis functions into two mutually disjoint subsets such that

$$
\hat{F}_{h}=\hat{F}_{f} \cup \hat{F}_{v}
$$

where the subscripts $f$ and $v$ denote the faces and volume of the reference element respectively. For 2-forms, locality implies that the face basis functions will have non-vanishing normal components along one and only one face while the volume basis functions will have no normal components along any of the the faces. The interpolatory face basis functions of polynomial degree $p$ are given by

$$
\hat{F}_{f}=\left\{\begin{array}{l}
L_{i}^{p}(\hat{x}) L_{j}^{p-1}(\hat{y}) L_{k}^{p-1}(\hat{z}) \hat{\mathbf{X}} \\
L_{i}^{p}(\hat{y}) L_{j}^{p-1}(\hat{x}) L_{k}^{p-1}(\hat{z}) \hat{\mathbf{Y}} \\
L_{i}^{p}(\hat{z}) L_{j}^{p-1}(\hat{x}) L_{k}^{p-1}(\hat{y}) \hat{\mathbf{Z}}
\end{array} \quad i=0, p ; j, k=0, \ldots, p-1\right.
$$

This set of functions is grouped into three sub-sets, one for each of the covariant basis vectors. The index $i$ loops over the 2 faces that are normal to these basis vectors. The indices $j$ and $k$ loop over the $p^{2}$ basis functions per face for a total of $6 p^{2}$. Finally, there will be a total of $3 p^{2}(p-1)$ interpolatory basis functions that are internal to the reference element given by

$$
\hat{F}_{v}=\left\{\begin{array}{l}
L_{i}^{p}(\hat{x}) L_{j}^{p-1}(\hat{y}) L_{k}^{p-1}(\hat{z}) \hat{\mathbf{X}} \\
L_{i}^{p}(\hat{y}) L_{j}^{p-1}(\hat{x}) L_{k}^{p-1}(\hat{z}) \hat{\mathbf{Y}} \\
L_{i}^{p}(\hat{z}) L_{j}^{p-1}(\hat{x}) L_{k}^{p-1}(\hat{y}) \hat{\mathbf{Z}}
\end{array}\right.
$$




\subsubsection{Basis Function Transformation Rules}

The basis functions presented are defined with respect to the reference coordinate system. In order to maintain coordinate independence, all properties of the functions defined in the reference coordinate system must be preserved under a transformation to a new global coordinate system, this property is known as invariance [26]. Table 1 gives the precise transformation rules for 1-forms and 2-forms, their respective exterior derivatives and the units of these transformations; all of which can be derived using the calculus of differential forms. The symbol $m$ denotes an arbitrary metric of distance while the symbol $\circ$ denotes composition. Figure 1 and Figure 2 give some visual examples of these transformations applied to particular members of the 1-form and 2-form bases of polynomial degree $p=1$. Each of the basis functions are plotted over three different elements corresponding to three different local to global mappings of geometry order $s=0$ (i.e. the reference element), $s=1$ and $s=2$. Note that our approach of defining all basis functions with respect to a reference (or local) coordinate system and later transforming the results to global element coordinate systems as necessary is very different than the approach presented in [23] where all basis functions are explicitly defined with respect to the barycentric coordinates of the global element. In Section 4.7 we show how our approach can lead to a computationaly efficient method for constructing the global matrices of (10).

Table 1

\begin{tabular}{llc}
\hline Object & Transformation Rule & Units \\
\hline \hline 1-form functions & $\mathbf{w} \circ \Phi=J^{-1} \hat{\mathbf{w}}$ & $m^{-1}$ \\
Curl of 1-form & $(\nabla \times \mathbf{w}) \circ \Phi=\frac{1}{|J|} J^{T}(\nabla \times \hat{\mathbf{w}})$ & $m^{-2}$ \\
2-form functions & $\mathbf{f} \circ \Phi=\frac{1}{|J|} J^{T} \hat{\mathbf{f}}$ & $m^{-2}$ \\
Div of 2-form & $(\nabla \cdot \mathbf{f}) \circ \Phi=\frac{1}{|J|}(\nabla \cdot \hat{\mathbf{f}})$ & $m^{-3}$ \\
\hline
\end{tabular}

Transformation Rules
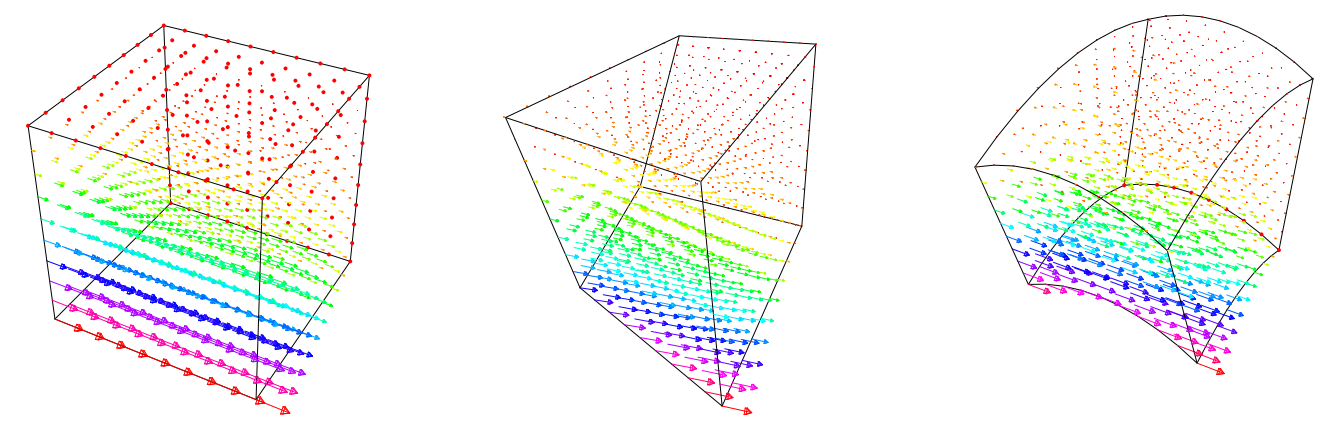

Fig. 1. Examples of a 1-form basis function transformation for elements of geometry order $s=0,1$ and 2 (left to right). 

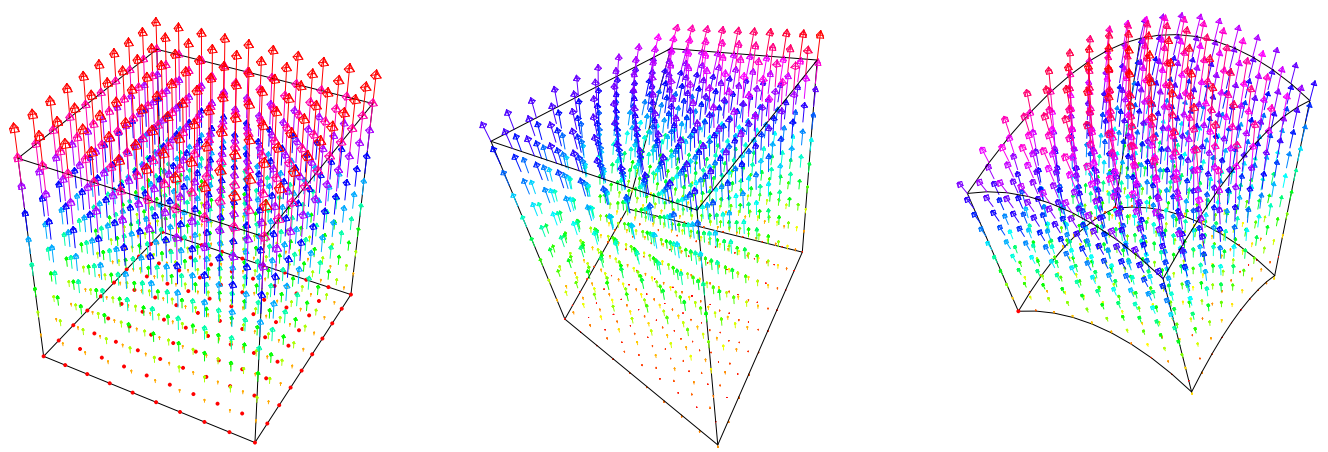

Fig. 2. Examples of a 2-form basis function transformation for elements of geometry order $s=0,1$ and 2 (left to right).

\section{$4.4 \mathcal{A}$ - Degrees of Freedom}

In [25], a set of integral based degrees of freedom (DOF) are presented which are presumed to be computed exactly. In practice, such DOF can be computationally expensive to implement and cannot always be integrated exactly using numerical quadrature. As such, we present a set of discrete DOF that are based on evaluation of a function at a point. These point based DOF satisfy the properties of invariance and locality and given a set of basis functions, they can be used to enforce unisolvence. The following DOF will always carry the physical units of the field that are not related to space (such as voltage, current, charge, etc ...) and will always be scale invariant.

Let $X$ denote a set of $p+1$ interpolation points over the unit interval $[0,1]$ and $X^{\prime}$ denote a set of $p$ interpolation points over the same interval. Furthermore, for the special case of $p=1$, let $X^{\prime}=\frac{1}{2}$. Now consider an arbitrary vector function $\mathbf{g}$, the set of linear functionals that make up the 1 -form point DOF are given by

$$
\mathcal{A}^{1}(\mathbf{g})=\left\{\begin{array}{l}
\mathbf{g}\left(\Phi\left(X_{i}^{\prime}, X_{j}, X_{k}\right)\right) \cdot J^{T} \hat{\mathbf{x}} \\
\mathbf{g}\left(\Phi\left(X_{k}, X_{i}^{\prime}, X_{j}\right)\right) \cdot J^{T} \hat{\mathbf{y}} \quad i=0, \ldots, p-1 ; j, k=0, \ldots, p \\
\mathbf{g}\left(\Phi\left(X_{j}, X_{k}, X_{i}^{\prime}\right)\right) \cdot J^{T} \hat{\mathbf{z}}
\end{array}\right.
$$

where $\hat{\mathbf{x}}, \hat{\mathbf{y}}$ and $\hat{\mathbf{z}}$ denote the contravariant basis vectors on the reference element. The set of linear functionals that make up the 2-form point DOF are given by

$$
\mathcal{A}^{2}(\mathbf{g})=\left\{\begin{array}{l}
\mathbf{g}\left(\Phi\left(X_{i}, X_{j}^{\prime}, X_{k}^{\prime}\right)\right) \cdot|J| J^{-1} \hat{\mathbf{X}} \\
\mathbf{g}\left(\Phi\left(X_{k}^{\prime}, X_{i}, X_{j}^{\prime}\right)\right) \cdot|J| J^{-1} \hat{\mathbf{Y}} \quad i=0, \ldots, p ; j, k=0, \ldots, p-1 \\
\mathbf{g}\left(\Phi\left(X_{j}^{\prime}, X_{k}^{\prime}, X_{i}\right)\right) \cdot|J| J^{-1} \hat{\mathbf{Z}}
\end{array}\right.
$$


where $\hat{\mathbf{X}}, \hat{\mathbf{Y}}$ and $\hat{\mathbf{Z}}$ denote the covariant basis vectors on the reference element.

The unisolvence property for DOF requires that

$$
\begin{gathered}
\mathcal{A}_{i}^{1}\left(\mathbf{w}_{j}\right)=\delta_{i, j}, \quad \mathbf{w}_{j} \in W_{h} \\
\mathcal{A}_{i}^{2}\left(\mathbf{f}_{j}\right)=\delta_{i, j}, \mathbf{f}_{j} \in F_{h}
\end{gathered}
$$

This property must hold in order for basis function expansions of the form (8) to be valid. In order to enforce unisolvence for a given basis (for example, the 1-form basis $W$ ), we first construct the matrix

$$
V_{i, j}=\mathcal{A}_{i}^{1}\left(\mathbf{w}_{j}\right)
$$

This matrix forms a linear mapping that is similar to a Vandermonde matrix. Because the degrees of freedom are linear functionals, we can construct a new set of degrees of freedom, denoted $\tilde{\mathscr{A}}^{1}$, by the relation

$$
\tilde{\mathcal{A}}^{1}=\left(V^{-1}\right)^{T} \mathcal{A}^{1}
$$

Note that by construction, the basis functions of (16) and (20) will satisfy the unisolvence property of (25) (up to some permutation of the basis functions). The procedure of (27) is therefore trivial for this particular case. However, this general procedure is valid for any proper set of basis functions including hierarchical bases such as those of [30] and [31].

\subsection{Validation of Basis Function Expansions}

Now let the operators $\Pi^{1}$ and $\Pi^{2}$ denote basis function expansions over an element $\Sigma$ such that

$$
\begin{aligned}
& \Pi^{1}(\mathbf{E})=\sum_{i=1}^{\operatorname{dim}\left(W_{h}\right)} \mathcal{A}_{i}^{1}(\mathbf{E}) \mathbf{w}_{i} \\
& \Pi^{2}(\mathbf{B})=\sum_{i=1}^{\operatorname{dim}\left(F_{h}\right)} \mathcal{A}_{i}^{2}(\mathbf{B}) \mathbf{f}_{i}
\end{aligned}
$$

The error in basis function expansions of this form is such that [25]

$$
\begin{aligned}
\left\|\mathbf{E}-\Pi^{1}(\mathbf{E})\right\| & \leq c_{1} \Delta h^{p}|\mathbf{E}| \\
\left\|\mathbf{B}-\Pi^{2}(\mathbf{B})\right\| & \leq c_{2} \Delta h^{p}|\mathbf{B}|
\end{aligned}
$$


where $c_{1}$ and $c_{2}$ are scalar valued constants of proportionality, $\Delta h$ is the characteristic size (or volume) of the element and $p$ is the polynomial degree of the basis functions.

To validate the 1 -form basis functions of (16) we choose a vector valued test function that is "sufficiently smooth", non-polynomial and has a well defined curl. Specifically, we choose

$$
\mathbf{E}=\{\sin (z), \cos (x), \exp (y)\}
$$

We then generate basis function expansions of this function and compute the error of these expansions using the $L_{2}$ volume norm: $\|\mathbf{E}\|_{2}=\sqrt{\int_{\Sigma}(\mathbf{E} \cdot \mathbf{E})}$. Similarly, to validate the 2-form basis functions of (20) we choose a vector valued test function that has a well defined divergence

$$
\mathbf{B}=\{\sin (x), \cos (y), \exp (z)\}
$$

Figure 3 shows logarithmic plots of the error in the expansions of (32) and (33) using the 1-form and 2-form basis functions of (16) and (20) for 4 levels of $h$ refinement and 6 levels of $p$-refinement.
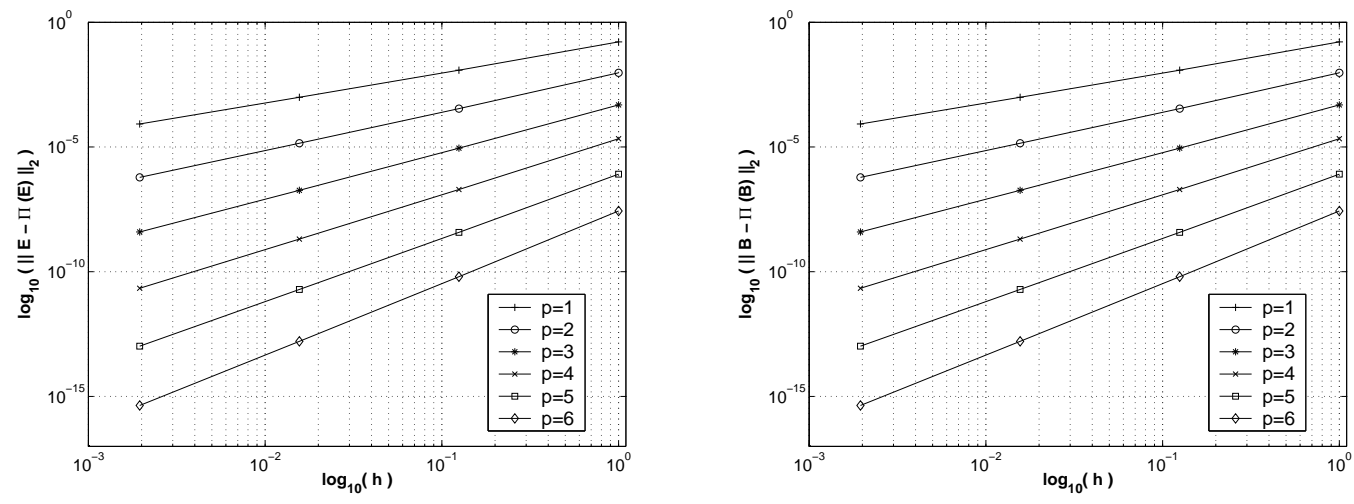

Fig. 3. Single element error $\left\|\mathbf{E}-\Pi^{1}(\mathbf{E})\right\|_{2}$ using 1-form basis functions (left) and $\left\|\mathbf{B}-\Pi^{2}(\mathbf{B})\right\|_{2}$ using 2-form basis functions (right) with 4 levels of $h$-refinement and 6 levels of $p$-refinement.

\subsection{Commuting Diagram Property}

The commuting diagram property states

$$
\nabla \times \Pi^{1}(\mathbf{E})=\Pi^{2}(\nabla \times \mathbf{E})
$$

The integral degrees of freedom of [25] satisfy this property exactly, meaning that the relation (34) is satisfied for any function $\mathbf{E}$. The discrete point degrees of free- 
dom of (23) and (24) satisfy this property in a discrete sense, meaning that as we increase the polynomial degree of the basis function expansion, the error in (34) converges to zero (using the $L 2$ norm); the same result one would expect when using numerical quadrature in the evaluation of the exact integral based DOF of [25]. Likewise, if $\mathbf{E} \in W_{h}$, the discrete point degrees of freedom satisfy (34) exactly. Figure 4 gives an example of this using the function of (32). This property has no effect on the error convergence of the finite element solution of (1).

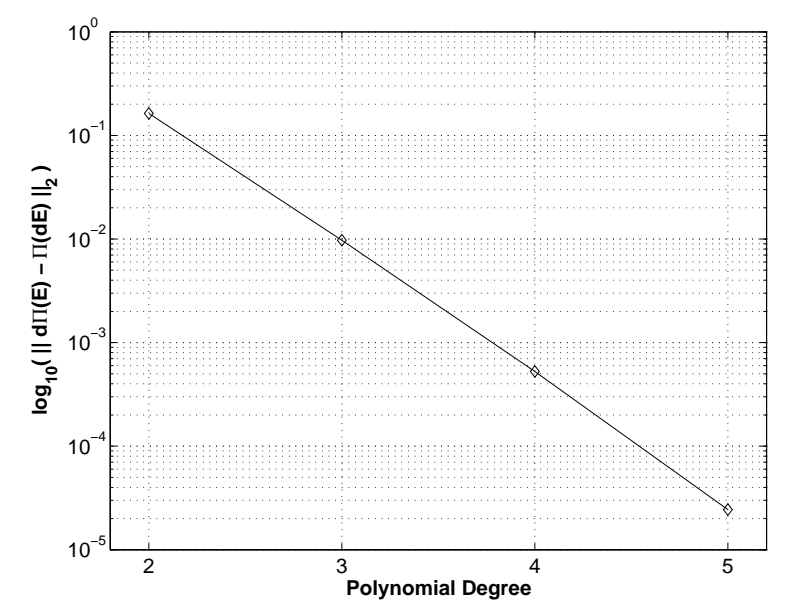

Fig. 4. Error in commuting diagram property using the discrete point degrees of freedom.

\subsection{Bilinear Forms}

In the Galerkin finite element procedure, we require bilinear forms to construct the system of linear equations of (10). We consider the general bilinear form

$$
M_{\tau}\langle\mathbf{g}, \mathbf{h}\rangle=\int_{\Omega} \tau \mathbf{g} \cdot \mathbf{h}
$$

where $\mathbf{g}$ and $\mathbf{h}$ are vector functions and $\tau$ is a symmetric positive definite (SPD) tensor function which can represent material properties such as electric and magnetic permeabilities and conductivities.

\subsubsection{Symmetric Bilinear Forms}

Now consider the symmetric bilinear form using the 1-form basis functions of Section 4.3. Using the properties of the local to global mapping (13), we re-write the bilinear form as follows 


$$
\begin{aligned}
M_{\tau}\left\langle\mathbf{w}_{i}, \mathbf{w}_{j}\right\rangle & =\int_{\Omega} \tau \mathbf{w}_{i} \cdot \mathbf{w}_{j} \\
& =\sum_{\Sigma \in \Sigma_{h}} \int_{\Sigma} \tau \mathbf{w}_{i} \cdot \mathbf{w}_{j} \\
& =\sum_{\Sigma \in \Sigma_{h}} \int_{\hat{\Sigma}}\left(\tau \mathbf{w}_{i} \cdot \mathbf{w}_{j}\right) \circ \Phi|J| \\
& =\sum_{\Sigma \in \Sigma_{h}} \int_{\hat{\Sigma}}\left(\tau \mathbf{w}_{i} \circ \Phi\right) \cdot\left(\mathbf{w}_{j} \circ \Phi\right)|J|
\end{aligned}
$$

Equation (36) shows that all calculations for the bilinear form can be performed on a standard reference element $\hat{\Sigma}$ by replacing the globally defined basis functions $\mathbf{w}$ with the appropriately transformed locally defined functions from Table 1 . This gives rise to a very computationally efficient algorithm for computing finite element approximations. For a given element topology and basis order,the basis functions only need to be computed once. Then, for every element of the same topology in the mesh, the results from the reference element can simply be mapped according to the transformation rules. This can significantly reduce computational time and storage requirements for a typical finite element computation.

Applying the transformation rules results in the following SPD bilinear form

$$
M_{\tau}\left\langle\mathbf{w}_{i}, \mathbf{w}_{j}\right\rangle=\int_{\hat{\Sigma}}\left((\tau \circ \Phi) J^{-1} \hat{\mathbf{w}}_{i}\right) \cdot\left(J^{-1} \hat{\mathbf{w}}_{j}\right)|J|
$$

The 1-form SPD mass matrix will have units of (or scale as) $\tau m^{1}$. Likewise, for 2-forms, we have the following SPD bilinear form

$$
M_{\tau}\left\langle\mathbf{f}_{i}, \mathbf{f}_{j}\right\rangle=\int_{\hat{\Sigma}}\left((\tau \circ \Phi) \frac{1}{|J|} J^{T} \hat{\mathbf{f}}_{i}\right) \cdot\left(\frac{1}{|J|} J^{T} \hat{\mathbf{f}}_{j}\right)|J|
$$

The 2-form SPD mass matrix will scale as $\tau m^{-1}$. These explicit bilinear forms can be used to assemble local (or single element) mass matrices used in the construction of the global linear system of (10).

\subsubsection{Discrete Curl Operator}

The coupled variational formulations of (4) and (5) have terms involving both 1form and 2-form functions; and will therefore require a mixed bilinear form. We can construct a rectangular matrix which maps discrete 1-forms to discrete 2-forms 
as follows

$$
T_{\tau}\left\langle\mathbf{w}_{i}, \mathbf{f}_{j}\right\rangle=\int_{\Sigma}\left(\tau \nabla \times \mathbf{w}_{i}\right) \cdot \mathbf{f}_{j}=M_{\tau}\left\langle\mathbf{f}_{i}, \mathbf{f}_{j}\right\rangle K_{i, j}
$$

resulting in a product of the 2-form mass matrix and a new matrix $K$ which we refer to as the topological derivative matrix. A topological derivative matrix for 1forms and 2-forms is a discrete version of the curl operator and is independent of the element geometry, i.e. it is an incidence map between the discrete differential 1form and 2-form degrees of freedom. Specifically, the topological derivative matrix is of the form

$$
K_{i, j}=\mathcal{A}_{i}^{2}\left(\nabla \times \mathbf{w}_{j}\right)
$$

where $\mathcal{A}_{i}^{2}$ are the 2 -form degrees of freedom from (24). In other words, we construct this matrix by projecting the curl of the 1-form basis functions from (16) onto the dual space of the 2-form degrees of freedom. Stated another way, we can write the curl of a 1-form as a linear combination of the 2-form basis functions. The resulting rectangular matrix contains only topological information and is independent of the mesh geometry (since the $J$ terms cancel out). It will have a number of rows equal to the dimension of the discrete 2-form basis and a number of columns equal to the dimension of the discrete 1 -form basis. For the case of first order basis functions (i.e. $p=1$ ), this matrix is the edge-face topological incident map commonly found in FDTD and FE methods, consisting of \pm 1 's and 0's [32]. Equation (40) is a generalization of this notion to higher-order basis functions.

\subsection{Global Assembly}

When assembling the global linear system of (10), it is imperative that all elements which share a sub-simplex (i.e. an edge or face) agree on the ordering and possibly direction of the basis vectors associated with that sub-simplex. In this section, we provide a method for ensuring that any two global elements which share an edge or face will agree on their orientation by introducing an orientation standard based on the global integer IDs of an element's primary vertices (i.e. for a hexahedron, these primary vertices are simply the 8 vertices that define the corners). Given this global standard, we demonstrate how to re-orient local edges and faces on an element in order to comply with this global standard. In this section, we will denote the global coordinate system using the generic variables $(u, v, w)$. 


\subsubsection{Edge Operations}

Here we define the symmetry operations of an edge. Consider an arbitrary line segment (or edge) defined by 2 generic integer IDs: $e=\{a, b\}$. We define the global $u$ orientation for this edge to be from the smallest integer ID to the largest integer ID. We now apply the global standard to a generic edge; there are only two cases to consider:

- Case 1 - E: Edge remains unchanged (Identity Operation)

$\operatorname{Min}(e)=a ; \quad e \mapsto\{a, b\}$

- Case 2 - !E: Edge is reversed

$\operatorname{Min}(e)=b ; \quad e \mapsto\{b, a\}$

At most, a local edge will have to be reversed during the global assembly process in order to comply with the global standard.

\subsubsection{Face Operations}

Here we define the symmetry operations of a face. Consider an arbitrary quadrilateral face defined by four generic integer IDs: $f=\{a, b, c, d\}$. We define the global $u$ orientation for this face to be from the smallest integer ID to its smallest neighbor (in a cyclical sense). The global $v$ orientation for this face is defined from the smallest integer ID to its largest neighbor (in a cyclical sense). We now apply the global standard to a generic face; there are 4 cases, each with 2 sub-cases, to consider for a total of 8 distinct possibilities:

- Case 1.1 - RO: Rotation of 0 degrees (Identity Operation) $\operatorname{Min}(f)=a$ and MinNeighbor $(a)=b ; \quad f \mapsto\{a, b, c, d\}$

- Case 1.2 - D2: Reflection about second diagonal $\operatorname{Min}(f)=a$ and MinNeighbor $(a)=d ; \quad f \mapsto\{a, d, c, b\}$

- Case 2.1 - R270: Rotation of 270 degrees $\operatorname{Min}(f)=b$ and MinNeighbor $(b)=c ; \quad f \mapsto\{b, c, d, a\}$

- Case 2.2 - $V$ : Reflection about vertical axis $\operatorname{Min}(f)=b$ and MinNeighbor $(b)=a ; \quad f \mapsto\{b, a, d, c\}$

- Case 3.1 - R180: Rotation of 180 degrees $\operatorname{Min}(f)=c$ and MinNeighbor $(c)=d ; \quad f \mapsto\{c, d, a, b\}$

- Case 3.2 - D1: Reflection about first diagonal $\operatorname{Min}(f)=c$ and MinNeighbor $(c)=b ; \quad f \mapsto\{c, b, a, d\}$

- Case 4.1 - R90: Rotation of 90 degrees $\operatorname{Min}(f)=d$ and MinNeighbor $(d)=a ; \quad f \mapsto\{d, a, b, c\}$

- Case 4.2 - $H$ : Reflection about horizontal axis $\operatorname{Min}(f)=d$ and MinNeighbor $(d)=c ; \quad f \mapsto\{d, c, b, a\}$ 
These possibilities represent the 8 different symmetry operations for a square, consisting of 4 rotations and 4 reflections [33]. During the global assembly process, a local face may need to be rotated or reflected in order to comply with the global standard. The edge and face symmetry operations are summarized in Figure 5.
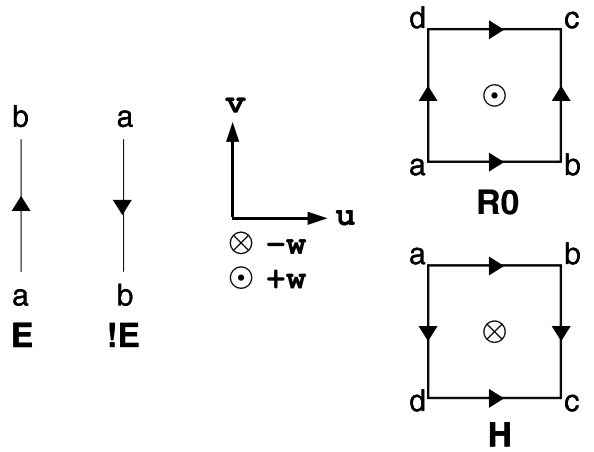

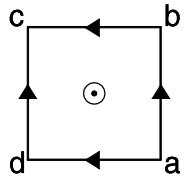

R90

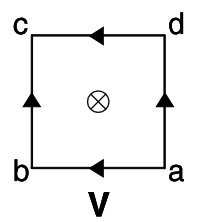

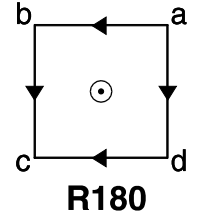

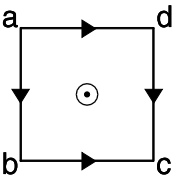

R270
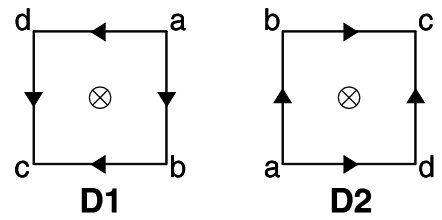

Fig. 5. Symmetry operations for edges and faces.

\subsubsection{Permutations}

Having defined all of the possible edge and face operations, we can now describe a permutation process for a single element. This process will take the global IDs of an arbitrary element and construct the local edge and face connections according to whatever local standard has been adopted. It will then check each local edge and face against the global standard and apply the necessary edge and face operations to enforce compliance. As an example, consider a 1 -form interpolatory face basis of degree $p=3$. In this case we will have 12 basis functions per face, 6 tangent to the local $u$ direction and 6 tangent to the local $v$ direction. Suppose two elements share a face designated by the 4 global integer IDs 2,5,8 and 11. Furthermore, suppose that in the first element, the face has the local orientation $f_{1}=\{11,8,2,5\}$ while in the second element it has the local orientation $f_{2}=\{5,2,8,11\}$. If we apply our standard to this face then the global orientation will be $f=\{2,5,11,8\}$ where the IDs $\{2,5\}$ define the local $u$ direction and the IDs $\{2,8\}$ define the local $v$ direction. In order to comply with this global standard we must apply a rotation of 180 degrees to $f_{1}$ and a vertical reflection to $f_{2}$. Figure 6 gives a visual example of this process. If we label the local face basis functions (using generic IDs) with respect to the global orientation as $w=$ $\{a, b, c, d, e, f, g, h, i, j, k, l\}$, then the face basis functions associated with the first element will be sorted as $w_{1} \mapsto\{-f,-e,-d,-c,-b,-a,-l,-k,-j,-i,-h,-g\}$ while the face basis functions associated with the second element will be sorted as $w_{2} \mapsto\{-c,-b,-a,-f,-e,-d, j, k, l, g, h, i\}$. The negative sign indicates that the local basis vector has changed sign as a result of the reorientation process. 

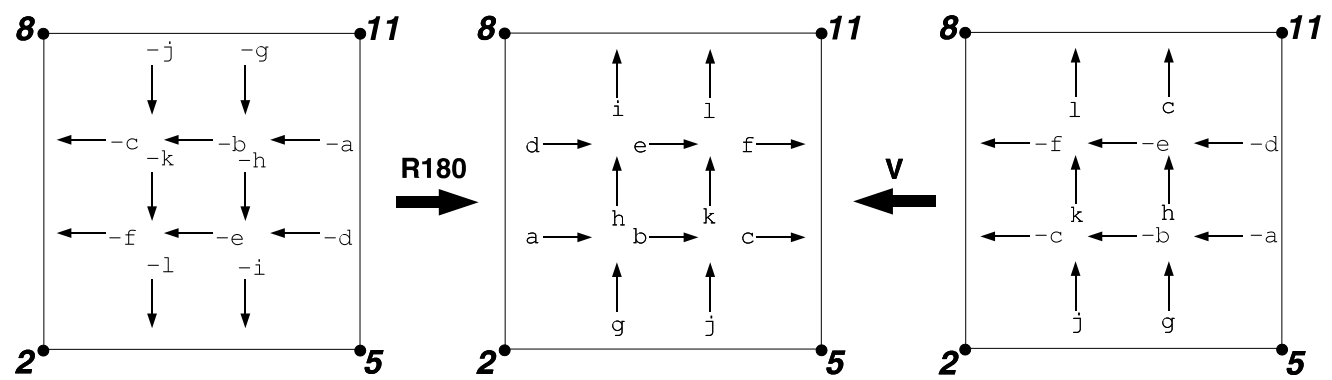

Fig. 6. Permutation process applied to 1-form interpolatory face basis functions of polynomial degree $p=3$. The global standard is displayed in the middle.

\section{High Order Temporal Discretization}

Having defined all of the necessary components for assembling the global linear system of ODEs of (10), we are now ready to discuss numerical methods to integrate this system in time. We will also discuss the nature of numerical energy and charge conservation.

\subsection{Time Integration and Numerical Stability}

To introduce the temporal discretization process, consider the second order leapfrog method applied to (10) for the special case of $\sigma=0$ (i.e. no dissipation) and $\mathbf{J}=0$ (i.e. no source term). This results in the following explicit update scheme

$$
\begin{aligned}
& e_{n+1}=e_{n}+\Delta t M_{\varepsilon}^{-1} K^{T} M_{\mu} b_{n} \\
& b_{n+1}=b_{n}-\Delta t K e_{n+1}
\end{aligned}
$$

where $\Delta t$ is the discrete time step. Rewriting this system in matrix form yields

$$
\left[\begin{array}{l}
e_{n+1} \\
b_{n+1}
\end{array}\right]=\left[\begin{array}{cc}
I & \Delta t M_{\varepsilon}^{-1} K^{T} M_{\mu} \\
-\Delta t K & I-\Delta t^{2} K M_{\varepsilon}^{-1} K^{T} M_{\mu}
\end{array}\right]\left[\begin{array}{l}
e_{n} \\
b_{n}
\end{array}\right]
$$

where $I$ is the identity matrix. More generically, we can write the system as

$$
\left[\begin{array}{c}
e_{n+1} \\
b_{n+1}
\end{array}\right]=Q\left[\begin{array}{l}
e_{n} \\
b_{n}
\end{array}\right]
$$


where the matrix $Q$ is called the amplification matrix. Stability of the method requires that [34]

$$
\|Q\|_{2} \leq 1+O(\Delta t)
$$

It can be shown that the amplification matrix $Q$ of (41) is similar to the matrix

$$
\tilde{Q}=\left[\begin{array}{rr}
I+R R^{T} & R \\
-R^{T} & I
\end{array}\right]
$$

Furthermore, it can be shown that the eigenvectors of the amplification matrix $\tilde{Q}$ form a complete eigenbasis. Therefore, a necessary and sufficient condition for satisfying (43) is given by

$$
\rho(Q) \leq 1
$$

where $\rho(Q)$ denotes the spectral radius of the amplification matrix $Q$. Following a similar approach to that of [27], it can be shown that a sufficient condition for satisfying (45) is given by the relation

$$
\Delta t \leq \frac{2}{\sqrt{\rho\left(K M_{\varepsilon}^{-1} K^{T} M_{\mu}\right)}}
$$

The stability condition of (46) is valid for all values of $p$, the order of the polynomial basis functions. However, as $p$ is increased, the value of $\rho\left(M_{\varepsilon}^{-1} K^{T} M_{\mu} K\right)$ will grow, thus requiring a smaller time step $\Delta t$.

\subsection{Conservation of Numerical Energy}

For an electromagnetic problem with no physical dissipation due to conductivity or absorbing boundary conditions the total electromagnetic energy should remain constant. In this particular mixed finite element method the instantaneous energy is the numerical version of the total energy given by

$$
\mathcal{E}=e^{T} M_{\varepsilon} e+b^{T} M_{\mu} b
$$

Many time integration methods such a forward Euler, backward Euler, RungeKutta, Adams-Bashforth, etc. are inherently dissipative and the energy as measured by (47) is not conserved; given an initial condition the electromagnetic energy will decay exponentially. 
The dissipative properties of a finite difference time integration scheme are based on the norm of the amplification matrix $Q$. There are three cases to consider

$$
\|Q\|_{2} \begin{cases}>1, & \text { unstable } \\ =1, & \text { neutrally stable (non-dissipative) } \\ <1, & \text { stable, dissipative }\end{cases}
$$

When the eigenvalues of the amplification matrix all lie within the unit circle in the complex plane, the method will be stable and dissipative. Non-dissipative methods have the additional property that the eigenvalues of the update matrix all lie on the unit circle in the complex plane, with additional constraints on the eignevectors for stability [27]. As demonstrated in [28], symplectic integration methods satisfy

$$
\|Q\|_{2}=1+\Delta t^{k} \cos (\gamma t)
$$

This implies that that the numerical energy of (47) is conserved in a time average sense; i.e. it oscillates periodically about the exact value and the amplitude of this oscillation is determined by the discrete time step $\Delta t$ and the order of the integration method $k$.

\subsection{Conservation of Numerical Charge}

Conservation of electric and magnetic charge is satisfied provided that (2) is satisfied for all time. The discrete electric field is expressed as a linear combination of 1-form basis functions. As such, the element to element interfaces do not have normal continuity and the electric field is not divergence free in the classical differential sense. Rather the field is divergence free only in the variational sense. This is required to allow for discontinuity of normal components across material interfaces. Introducing a scalar valued, piecewise continuous 0 -form trial function $\phi^{\prime}$, the variational form of the electric charge constraint of (2) is

$$
\int_{\Omega} \nabla \cdot(\varepsilon \mathbf{E}) \phi^{\prime}=-\int_{\Omega} \varepsilon \mathbf{E} \cdot \nabla \phi^{\prime}+\int_{\partial \Omega}\left(\varepsilon \mathbf{E} \phi^{\prime}\right) \cdot \hat{\mathbf{n}}=0
$$

Since the electric field is not required to be divergence free on the external boundary, we choose $\phi=0$ on $\partial \Omega$ yielding the constraint

$$
\int_{\Omega} \varepsilon \mathbf{E} \cdot \nabla \phi^{\prime}=0
$$


A discrete matrix version of this has the form [35]

$$
G^{T} M_{\varepsilon} e=0 \forall e \in \mathcal{A}^{1}(\mathbf{E})
$$

where the rectangular matrix $G$ is a discrete version of the gradient operator; similar in construction to the discrete curl operator of (40), except now it is an incidence map between 0 -form and 1-form degrees of freedom (i.e. for first order basis functions, this matrix is the standard node-edge topological incidence map). Likewise, a discrete version for the conservation of magnetic charge is given by

$$
D b=0 \forall b \in \mathcal{A}^{2}(\mathbf{B})
$$

where the rectangular matrix $D$ is a discrete version of the divergence operator; again, similar in construction to the discrete curl operator of (40), except now it is an incidence map between 2-form and 3-form degrees of freedom (i.e. for first order basis functions, this matrix is the standard face-cell topological incidence map).

\subsection{Higher Order Conservative Methods}

The leap-frog method of (41) is second order accurate in time. Higher order conservative methods exist such as those of [36] and [37]; which were originally derived for Hamiltonian systems with applications in astrophysics and molecular dynamics. In [28], these symplectic methods are applied to finite element discretizations of Maxwell's equations of the form (10) using a general symplectic algorithm. In Algorithm 1, we present the inputs, procedure and outputs of the general high order symplectic integration algorithm. In general, a method of order $k$ will require $k$ evaluations of the functions $F$ and $G$. Therefore, as the order of the method is increased the overall computational costs will increase likewise. However, as shown in [28], higher order time integration methods can yield drastic improvement's in accuracy for roughly the same computational cost as standard low order methods. The order of the method can be adjusted simply by providing the algorithm with a corresponding set of coefficients, $\alpha$ and $\beta$, each of length order. Table 2 lists exact values of the sets of coefficients for methods of order 1 through 4 , as originally computed by [36] and [37]. Note that for the leap-frog method, $\alpha=1$ and $\beta=1$.

For example, the third order symplectic integration method from Table 2 applied to (10) can be written in matrix form as

$$
\left[\begin{array}{c}
e_{n+1} \\
b_{n+1}
\end{array}\right]=Q_{3} Q_{2} Q_{1}\left[\begin{array}{l}
e_{n} \\
b_{n}
\end{array}\right]
$$


input : order, the order of the method

$F(e, b)$ and $G(b)$, two functions

$\alpha$ and $\beta$, two sets of coefficients

$F_{0}$ and $G_{0}$, the initial conditions

$t_{0}$ and $t_{f i n}$, initial and final time

$\Delta t$, the time step to use

output $\quad: e_{f i n}$ and $b_{f i n}$, the fields at time $t_{f i n}$

Compute the number of time steps:

nstep $=\frac{t_{\text {fin }}-t_{0}}{\Delta t}$

Set initial conditions:

$e_{1} \leftarrow F_{0}$

$b_{1} \leftarrow G_{0}$

Begin loop over time steps:

for $i=1$ to nstep do

Begin integration method update:

$e_{\text {in }} \leftarrow e_{i}$

$b_{\text {in }} \leftarrow b_{i}$

for $j=1$ to order do

Compute the update time for this step:

$t_{j}=i \Delta t+\sum_{k=1}^{j-1} \alpha_{k} \Delta t$

Update the field values:

$e_{\text {out }}=e_{\text {in }}+\beta_{j} \Delta t F\left(e_{i n}, t_{j}\right)$

$b_{\text {out }}=b_{\text {in }}+\alpha_{j} \Delta t G\left(b_{\text {out }}\right)$

$e_{\text {in }} \leftarrow e_{\text {out }}$

$b_{\text {in }} \leftarrow b_{\text {out }}$

end

Update field values for this time step:

$e_{i+1} \leftarrow e_{\text {out }}$

$b_{i+1} \leftarrow b_{\text {out }}$

end

$e_{\text {fin }} \leftarrow e_{\text {nstep }}+1$

$b_{\text {fin }} \leftarrow b_{\text {nstep }+1}$

Algorithm 1. General Symplectic Integration Algorithm

where the matrices $Q_{i}$ are of the form

$$
Q_{i}=\left[\begin{array}{cc}
I & \beta_{i} \Delta t M_{\varepsilon}^{-1} K^{T} M_{\mu} \\
-\alpha_{i} \Delta t K & I+\alpha_{i} \beta_{i} \Delta t^{2} K M_{\varepsilon}^{-1} K^{T} M_{\mu}
\end{array}\right]
$$

This results in a system amplification matrix $Q$ that is a product of the $Q_{i}$. Again, it can be shown that the product matrix $q$ is similar to product matrix whose eigenvectors form a complete eigenbasis. Therefore, a sufficient condition for stability 


\begin{tabular}{ll}
\hline \multicolumn{2}{l}{ Order $1-$ Truncation Error $=\Delta t^{2}$} \\
\hline$\alpha_{1}=1$ & $\beta_{1}=1$ \\
\hline Order 2 - Truncation Error $=\Delta t^{3}$ & \\
\hline$\alpha_{1}=1 / 2$ & $\beta_{1}=0$ \\
$\alpha_{2}=1 / 2$ & $\beta_{2}=1$ \\
\hline
\end{tabular}

\begin{tabular}{ll} 
Order $3-$ Truncation Error $=\Delta t^{4}$ & \\
\hline$\alpha_{1}=2 / 3$ & $\beta_{1}=7 / 24$ \\
$\alpha_{2}=-2 / 3$ & $\beta_{2}=3 / 4$ \\
$\alpha_{3}=1$ & $\beta_{3}=-1 / 24$ \\
\hline
\end{tabular}

Order 4 - Truncation Error $=\Delta t^{5}$

Table 2

\begin{tabular}{ll}
\hline$\alpha_{1}=\left(2+2^{1 / 3}+2^{-1 / 3}\right) / 6$ & $\beta_{1}=0$ \\
$\alpha_{2}=\left(1-2^{1 / 3}-2^{-1 / 3}\right) / 6$ & $\beta_{2}=1 /\left(2-2^{1 / 3}\right)$ \\
$\alpha_{3}=\left(1-2^{1 / 3}-2^{-1 / 3}\right) / 6$ & $\beta_{3}=1 /\left(1-2^{2 / 3}\right)$ \\
$\alpha_{4}=\left(2+2^{1 / 3}+2^{-1 / 3}\right) / 6$ & $\beta_{4}=1 /\left(2-2^{1 / 3}\right)$
\end{tabular}

Symplectic Integration Coefficients for Methods of Order 1 Through 4 of this third order update method is

$$
\rho\left(Q_{i}\right) \leq 1 ; \quad i=1,2,3
$$

\section{Computational Examples}

\subsection{Time Domain Resonant Cavity Analysis}

In these experiments, we compute the resonant modes of two different cavity geometries by directly solving the time dependent PDE of (1) subject to a PEC boundary condition. We begin by creating an oscillating electromagnetic field inside the cavity by applying a time dependent, vector valued current source to a random sampling of the interior DOF of the spatially discretized PDE of (10). The simple current source has a temporal profile equal to the first derivative of a Gaussian pulse. In addition, the pulse is randomly oriented each time it is applied to a degree of freedom; this is done to ensure that all of the modes of the cavity are excited. We then discretize this problem in time using the first order symplectic (or leap-frog) method from [28] and use a standard inverse power method to compute the largest stable time step as dictated by the stability condition of (46). A diagonally scaled 
conjugate gradient algorithm is used to invert the mass matrix at every time step. Setting the speed of light equal to unity, we let the simulation run for a physical time of 200 seconds. Upon completion, we extract the time dependent values from the discrete 1-from solution vector, a discrete version of the voltage, and Fourier transform the result to obtain both the transverse electric (TE) and transverse magnetic (TM) resonant modes of the cavity. We then compare the computed modes with their known exact values.

\subsubsection{Cubic Cavity}

We begin with the simple cavity geometry of a unit cube. The computational mesh for this problem consists of a relatively coarse $8 \times 8 \times 8$ series of hexahedral elements. The exact TE and TM resonant modes for a cube of this geometry are given by [38]

$$
f_{l, m, n}=\frac{1}{2 \pi} \sqrt{\pi^{2}\left(l^{2}+m^{2}+n^{2}\right)} \text { for } l, m=1, \ldots ; n=0, \ldots
$$

The longer the simulation is run in time, the more accurately we can resolve the peaks of the resulting Fourier spectrum; we choose a physical time of 200 seconds which yields reasonably sharp "spikes" in the frequency domain.

In Table 3 we summarize the results of three different resonant cavity calculations on the same mesh, using basis functions of polynomial degree $p=1,2$ and 3 . In Figure 7, Figure 8 and Figure 9 we show the respective computed Fourier spectrum for the first 27 resonant frequencies of the cavity. The vertical lines in these plots represent the locations of the exact resonant modes. Note that the vertical scale of these plots is essentially irrelevant, the height of each peak is simply a relative measure of how much this particular mode was exited by the random sampling process. Note that for the $p=1$ case, the computed high-frequency modes are drastically "up-shifted," falling far short of their exact values. This is due the coarse nature of the mesh and the low order of approximation. As shown in Table 3, the computed error in all three cases increases as the frequency of the mode increases. However, the higher order methods yield a much slower rate of growth with an overall error that is orders of magnitude smaller than the standard $p=1$ method. As demonstrated in [28], the use of higher order symplectic integration methods can yield even higher levels of accuracy. In addition to this, it is important to note that for all three cases presented, there are no "spurious" modes (i.e. non-physical resonant modes) or late time instabilities.

\subsubsection{Spherical Cavity}

In this experiment we compute the resonant modes of a spherical cavity using two different meshes: a very fine mesh (Figure 10) with a relatively small $\Delta h$ value 


\begin{tabular}{l|rrr}
\hline & $p=1$ & $p=2$ & $p=3$ \\
\hline \hline No. Unknowns & 1,194 & 13,872 & 45,000 \\
Abs. Error in 5th Mode & 0.03728 & 0.00104 & 0.00011 \\
Abs. Error in 15th Mode & 0.19809 & 0.00850 & 0.00049 \\
Abs. Error in 25th Mode & 0.36144 & 0.02269 & 0.00247 \\
\hline
\end{tabular}

Table 3

Summary of cubic cavity results

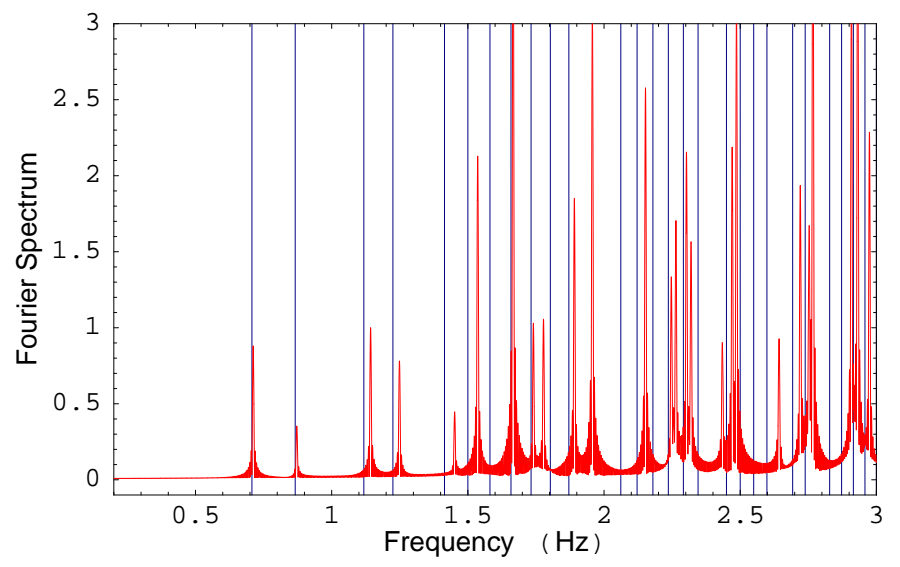

Fig. 7. Computed resonant modes of cubic cavity using basis functions of degree $p=1$

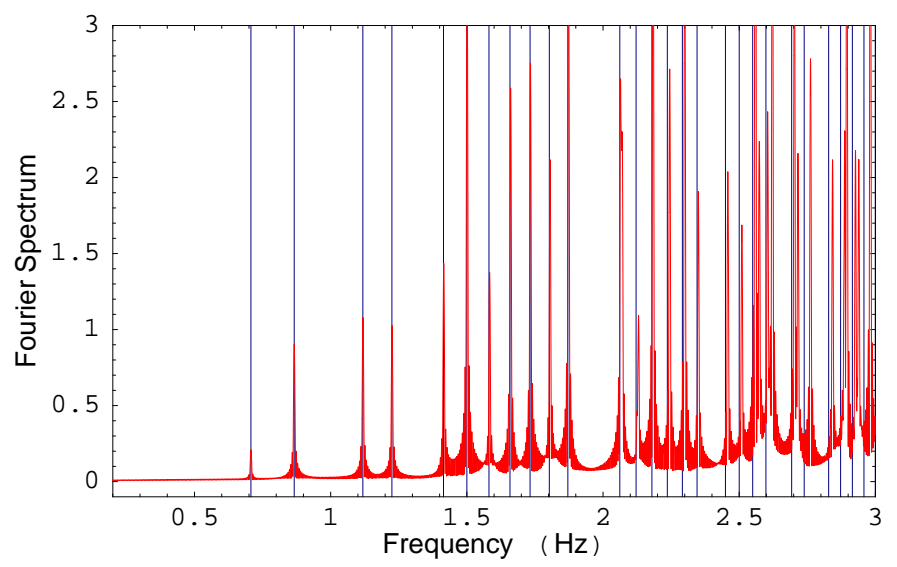

Fig. 8. Computed resonant modes of cubic cavity using basis functions of degree $p=2$

and a very coarse mesh (Figure 11) with a large $\Delta h$ value. The fine mesh will use standard first order geometry elements $(s=1)$ while the coarse mesh will make use of curved surface elements $(s=2)$. Use of curved elements on the surface allow the mesh to be very coarse while still accurately modeling the geometric properties of the spherical surface.

Figure 12 and Figure 13 show the results of two separate calculations, one demonstrating $h$-Refinement using a discrete basis of polynomial degree $p=1$ on the fine mesh and the other demonstrating $p$-Refinement using a discrete basis of polyno- 


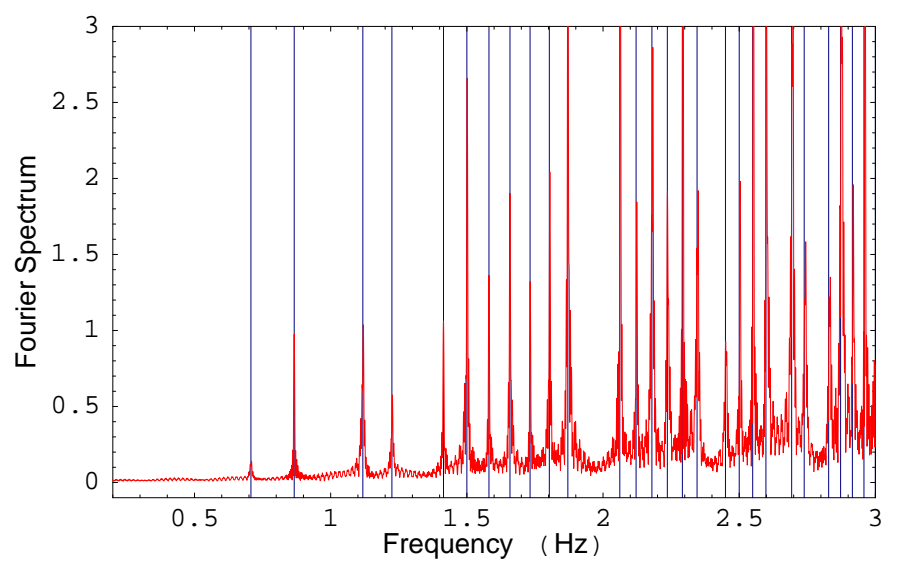

Fig. 9. Computed resonant modes of cubic cavity using basis functions of degree $p=3$

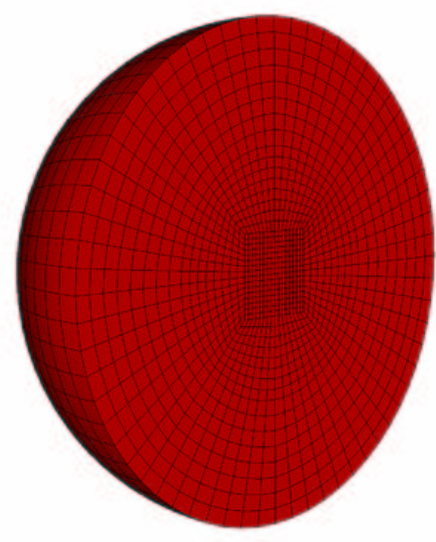

Fig. 10. Cross section of $h$-Refined spherical mesh .

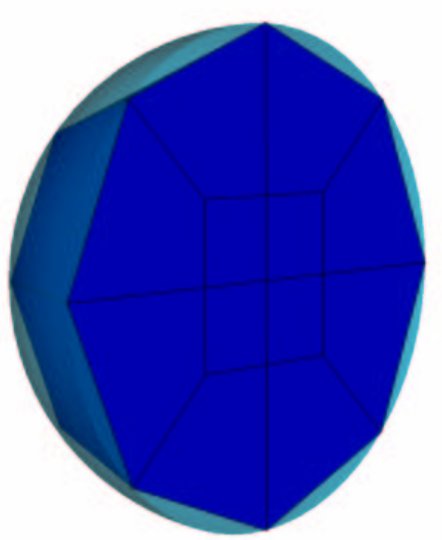

Fig. 11. Cross section of coarse spherical mesh with curvilinear surface elements

mial degree $p=3$ on the very coarse mesh with curvilinear surface elements. The results of these two calculations are summarized in Table 4. Qualitatively speaking, both simulations yield the same results; however, the computational costs are strikingly different. For example, to achieve a prescribed error tolerance of $10^{-3}$ in the first computed mode, using a $p$-Refinement method runs 42 times faster than a corresponding $h$-Refinement method.

\subsection{Guided Wave Analysis}

In these computational examples we simulate the propagation of an EM wave in two different guiding structures: a coaxial cable and a single mode optical fiber. In addition, we investigate the numerical dispersion properties of the method via example. It is well known that higher order methods are better at reducing the effects of numerical dispersion over standard first order $h$-refined methods [12], [13], [14], [15]. For the specific case of the second order accurate leap frog method 


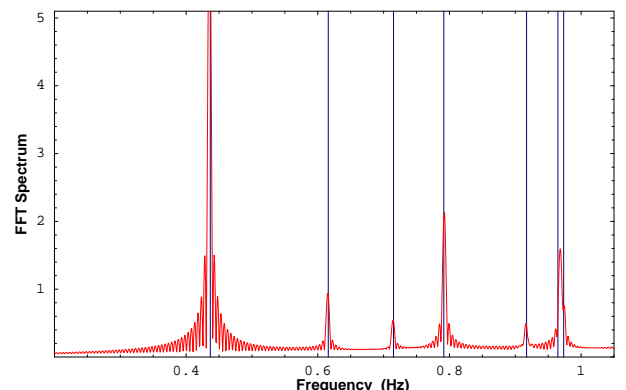

Fig. 12. Computed resonant modes of spherical cavity using $h$-Refinement.

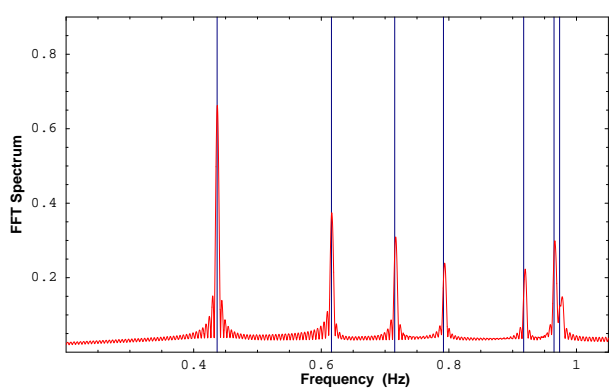

Fig. 13. Computed resonant modes of spherical cavity using $p$-Refinement.

\begin{tabular}{l|rr}
\hline & $h$-Refinement & $p$-Refinement \\
\hline \hline Physical Time & $200 \mathrm{sec}$ & $200 \mathrm{sec}$ \\
Error Tol. for 1st Mode & $1 \mathrm{e}-3$ & $1 \mathrm{e}-3$ \\
Abs. Error in 1st Mode & $7.167 \mathrm{e}-4$ & $4.431 \mathrm{e}-4$ \\
No. Elements & 28,672 & 32 \\
No. Unknowns & 87,632 & 2,832 \\
No. Nonzeros & $2,849,360$ & 615,888 \\
Fill Ratio & $0.0371 \%$ & $7.679 \%$ \\
Largest Stable Time Step & $0.007 \mathrm{sec}$ & $0.03 \mathrm{sec}$ \\
Number of Steps & 28,572 & 6,668 \\
Avg. CPU time/step & $1.00649 \mathrm{sec}$ & $0.100927 \mathrm{sec}$ \\
Total Run Time & $\mathbf{4 7 9 . 3 ~} \mathbf{~ m i n}$ & $\mathbf{1 1 . 2} \mathbf{~ m i n}$ \\
\hline
\end{tabular}

Table 4

Comparison of computational cost for $h$-Refinement and $p$-Refinement

applied to time domain vector finite element solutions of Maxwell's equations (with the free space speed of light scaled to unity), the discrete dispersion relation is of the form

$$
\omega^{2}=\left(\frac{2 \pi}{\lambda}\right)^{2}\left(1+O\left(\left(\frac{\Delta h}{\lambda}\right)^{2 p}\right)+O\left(\left(\frac{\Delta t}{\lambda}\right)^{2}\right)\right)
$$

where $\lambda$ is the characteristic wavelength of the EM wave, $\omega$ is the characteristic frequency and $p$ is polynomial degree of the finite element basis functions. Thus, for a given characteristic element size $\Delta h$, an increase in the value $p$ will reduce the numerical dispersion error more than a corresponding level of $h$-refinement (i.e. for hexahedral elements: $\left.\Delta h \mapsto \frac{1}{8} \Delta h\right)$. 


\subsubsection{Coaxial Waveguide}

In this example we simulate the propagation of an EM wave along a coaxial waveguide. The problem is excited with a time dependent voltage source boundary condition applied to the input cap of the mesh. The voltage source has a temporal profile equal to a ramped sine wave function and a spatial profile proportional to the inverse of the radial coordinate. A PEC boundary condition is applied to the inner and outer cylindrical walls while an absorbing boundary condition (ABC) is applied to the end cap of the mesh. An analytic (or exact) solution to this problem exists and is simply the value of the time and space dependent voltage source at the input boundary evaluated at the retarded time $t^{\prime}=t-c / z$, where $c$ is the speed of light in the guide and $z$ is the propagation direction. This allows for a normed error analysis of the method, thus providing quantitative insight into the dispersion properties of the method. Scaling the speed of light equal to unity, we set the characteristic frequency of the voltage source to 0.788 while the mesh has a length of 100 units. This implies that at time $t=100$, there will be roughly 12 full wavelengths in the coaxial mesh. Due to numerical dispersion, the computed solution will gradually get out of phase with the exact solution.

Figure 14 shows two meshes of the coaxial waveguide, a fine mesh $(\Delta h)$ and a coarse mesh $(8 \Delta h)$. Figure 15 shows a magnitude plot of the computed electric field along with a sliced vector plot of the computed magnetic field. In Figure 16 we plot the maximum computed error as a function of the discrete time step for two different simulations: one using first order $(p=1)$ basis functions on the fine mesh and the other using second order $(p=2)$ basis functions on the coarse mesh with curvilinear surface elements $(s=2)$ on the inner and outer cylindrical walls. The error in the approximate electric field, $\delta=\mathbf{E}-\mathbf{E}_{h}$, is computed for each element in the mesh using the $L_{2}$ volume norm. Note that in both cases, the maximum global phase error due to numerical dispersion increases as a function of time, but the $p$-refined simulation yields a much slower rate of growth.
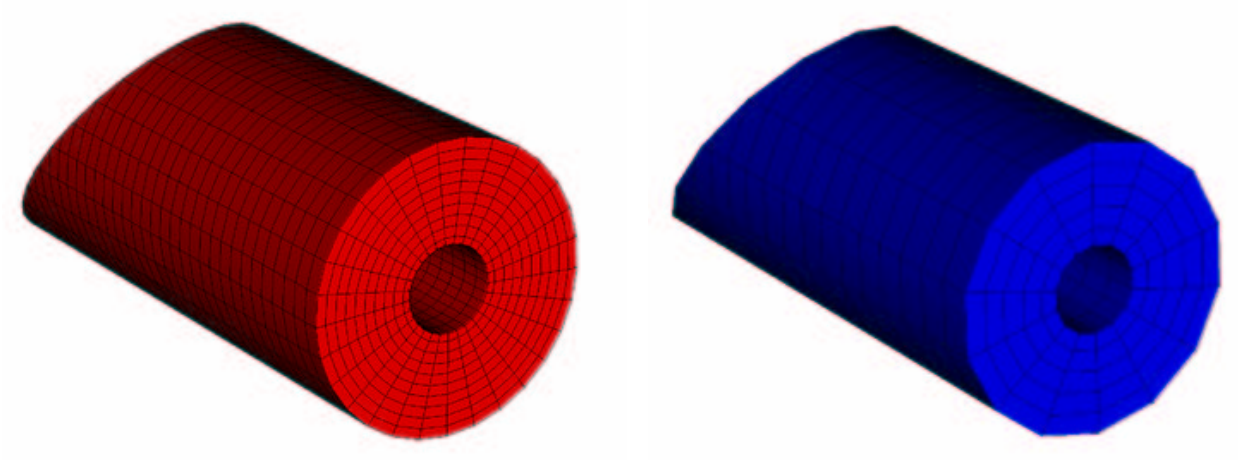

Fig. 14. Coarse and fine coaxial waveguide meshes.

In Figure 17 we plot the base $10 \log$ of the computed error as a function of propa- 


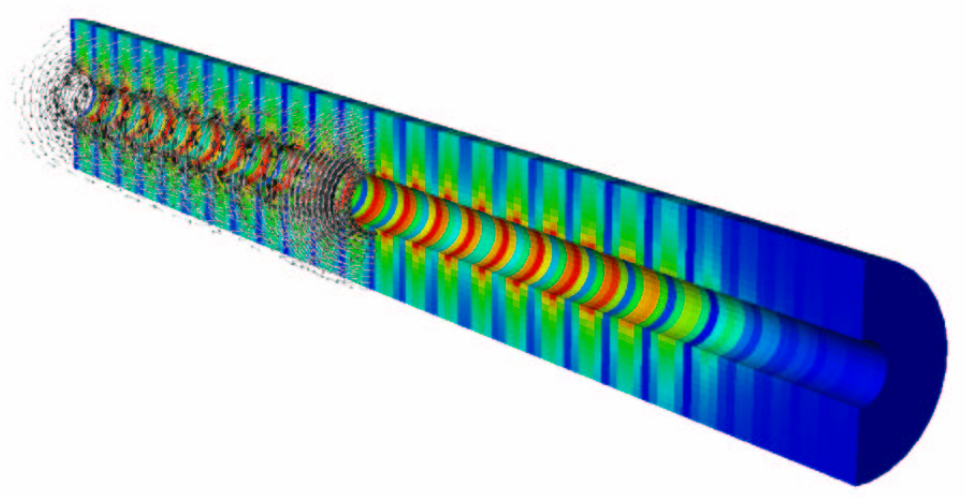

Fig. 15. Example of the computed electric and magnetic fields for the coaxial waveguide simulation.

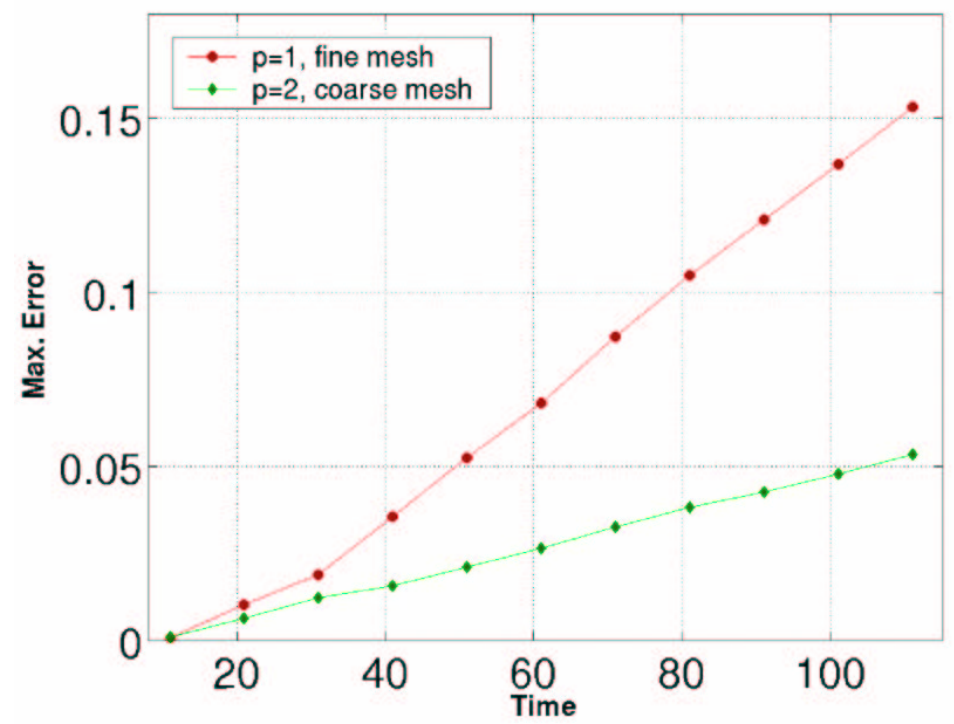

Fig. 16. Maximum phase error at each time step for coaxial waveguide simulation.

gation distance along the coarse mesh for a fixed time step value. We do this for the three cases $p=1,2$ and 3. Again, note that as $p$ is increased, the maximum value and the growth rate of the phase error due to numerical dispersion is drastically decreased. Also note that for the $p=1$ case, the phase error begins to decrease at around $z=60$; this is because the computed wave is now a full 180 degrees out of phase with the exact wave. It should be noted, the improved performance of $p$-refinement comes at a corresponding increase in computational cost: the total number of 1 -form problem unknowns for the $p=1$ case is 14,910 , for $p=2$ there are 111,692 unknowns and for $p=3$ there are 368,466 unknowns. 


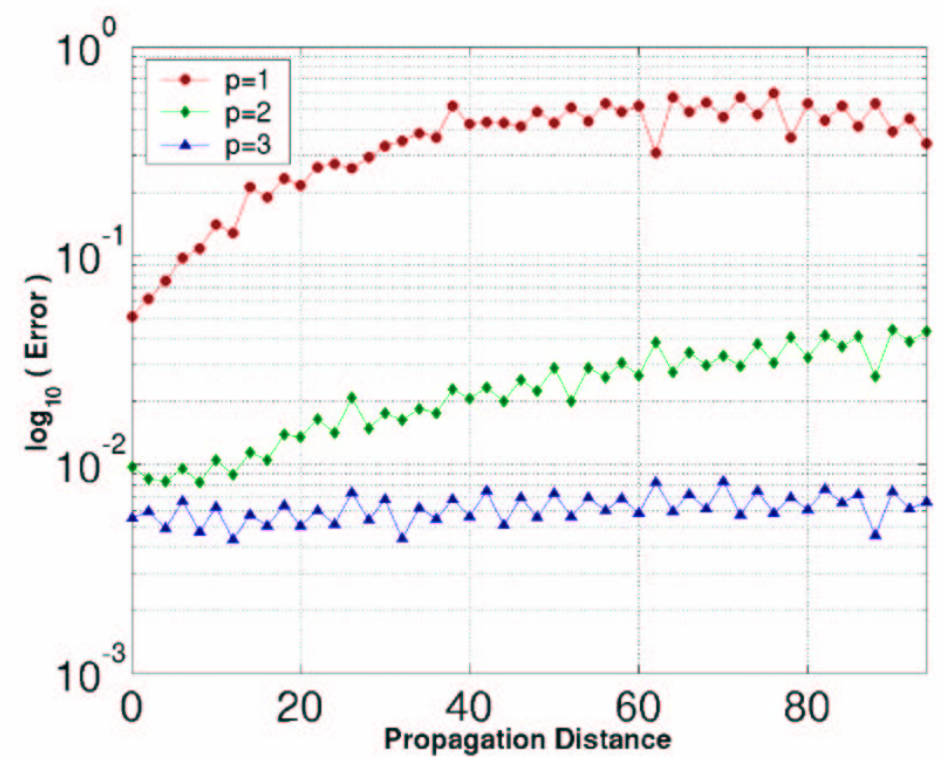

Fig. 17. Base $10 \log$ of computed phase error vs. propagation distance at fixed time for coaxial waveguide simulation.

\subsubsection{Fiber Optic Waveguide}

In this example we simulate the propagation of a TE01 mode along a $100 \mu \mathrm{m} \mathrm{sec-}$ tion of a single mode optical fiber using third order $(p=3)$ basis functions and curvilinear surface elements $(s=2)$ at the core and cladding surfaces. The core has a radius of $5 \mu \mathrm{m}$ while the cladding has a radius of $25 \mu \mathrm{m}$. The core index of refraction is 1.471 while the cladding index is 1.456 . With these properties, the fiber is capable of of propagating a $1550 \mathrm{~nm}$ optical wave. The problem is excited with a space and time dependent pulsed voltage source boundary condition applied to the input cap of the mesh. The spatial dependence of the voltage source is derived from Bessel functions of the first and second kind with the appropriate transverse propagation constants to satisfy continuity across the core / cladding interface while the temporal profile is a pulsed sine wave containing 20 wavelengths as shown in Figure 18. A PEC boundary condition is applied to the outer cladding surface while an absorbing boundary condition (ABC) is applied to the end cap of the mesh.

Use of $p=3$ basis functions and $s=2$ curvilinear surface elements permits the use of a relatively coarse mesh, namely 1 transverse element per wavelength instead of the usual 10 transverse elements for a standard low order method. For this simulation, the fiber optic mesh consists of only 8,208 elements. Standard cell-centered visualization methods for the fields result in a very coarse representation on such a mesh. Figure 19 shows a magnitude plot of the electric field vector at time step $t=0.187 p s$ sampled at 25 points per element, indicating the high degree of field resolution within in each element using high order basis functions. 

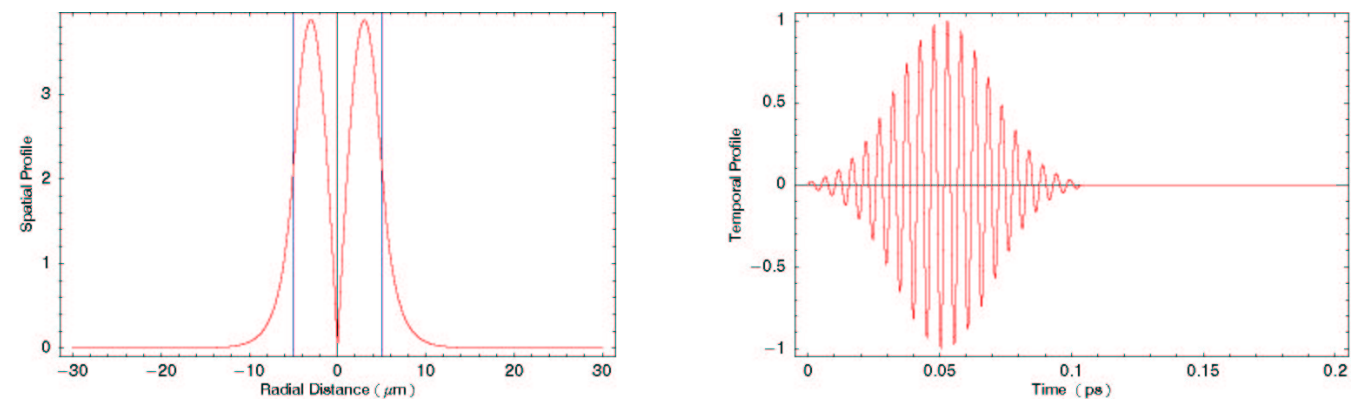

Fig. 18. Spatial and temporal profile of pulsed voltage source used to excite fiber optic simulation.

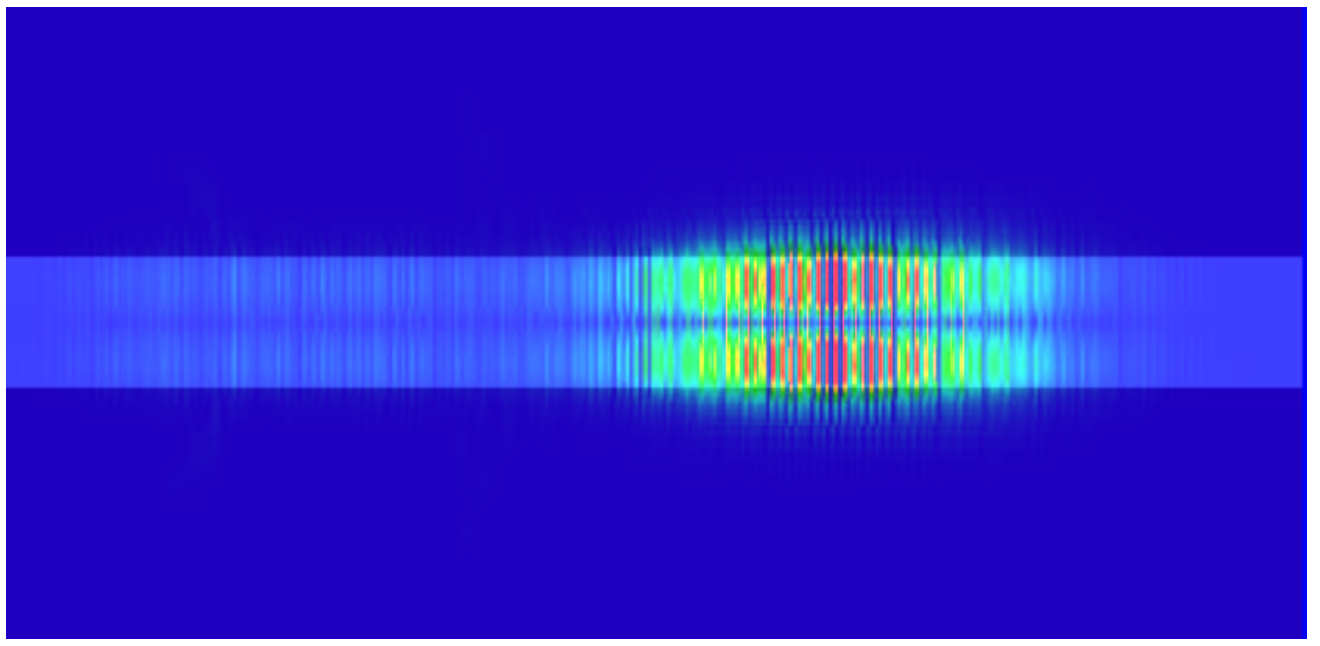

Fig. 19. Snapshot of electric field magnitude at $t=0.187 \mathrm{ps}$ in fiber optic simulation.

\section{Conclusions}

We have presented a high order mixed vector finite element method suitable for discretizing the time dependent Maxwell equations on unstructured grids that is of arbitrary order accuracy in space and up to 5th order accurate in time. The method is charge and energy conserving, conditionally stable and able to maintain the proper element to element continuities for the discrete electric and magnetic fields. For clarity and brevity, this work was presented for Hexahedral elements; however, many of the results of this paper can be applied to elements of other topologies (e.g. prisms, tetrahedrons, etc ...). We have demonstrated via computational example the benefits of the proposed method including the improved reduction of numerical dispersion error for electrically large problems.

\section{References}

[1] K. S. Yee. Numerical solution of initial boundary value problems involving Maxwell's equations in isotropic media. IEEE Trans. Ant. Prop., 14(3):302-307, 1966. 
[2] A. Taflove and M. E. Brodwin. Numerical solution of steady-state electromagnetic scattering problems using the time-dependent Maxwell's equations. IEEE Trans. Microwave Theory Tech., 23:623-630, 1975.

[3] G. Mur. Absorbing boundary conditions for the finite-difference approximation of the time-domain electromagnetic field equations. IEEE Trans. Electromagnetic Compatibility, 23(4):377-382, 1981.

[4] J. Berenger. A perfectly matched layer for the absorption of electromagnetic waves. J. Comput. Phys., 114(2):185-200, 1994.

[5] A. C. Cangellaris and D. B. Wright. Analysis of the numerical error caused by the stair-stepped approximation of a conducting boundary in FDTD simulations of electromagnetic phenomena. IEEE Trans. Ant. Prop., 39(10):1518-1525, 1991.

[6] R. Holland. Pitfalls of staircase meshing. IEEE Trans. Electromagnetic Compatibility, 35(4):434-439, 1993.

[7] N. Madsen and R. Ziolkowski. A 3 dimensional modified finite volume techniqe for Maxwell's equations. Electromagnetics, 10(1):147-161, 1990.

[8] R. Holland, V. Cable, and L. Wilson. Finite-volume time-domain techniques for EM scattering. IEEE Trans. Electromagnetic Compatibility, 33(4):281-294, 1991.

[9] N. K. Madsen. Divergence preserving discrete surface integral method for Maxwell's curl equations using non-orthogonal unstructured grids. J. Comput. Phys., 119(1):3445, 1995.

[10] D. A. White. Discrete Time Vector Finite Element Methods for Solving Maxwell's Equations on $3 D$ Unstructured Grids. PhD thesis, University of California at Davis, Livermore, California, 1997.

[11] P. Boullard A. Deraemaeker, I. Babuska. Dispersion error and pollution of the FEM solution for the Helmholtz equation in one, two, and three dimensions. 46(4):471-499, 1999.

[12] P. Monk and A. Parrot. A dispersion analysis of finite element methods for Maxwell's equations. SIAM J. Sci. Comp., 15(4):916-937, 1994.

[13] S. Warren and W. Scott. An investigation of numerical dispersion in the vector finite element method using quadrilateral elements. IEEE Trans. Ant. Prop., 42(11):15021508, 1994.

[14] S. Warren and W. Scott. Numerical dispersion in the finite element method using triangular edge elements. Opt. Tech. Lett., 9(6):315-319, 1995.

[15] M. Ainsworth and J. Coyle. Hierarchic $h p$-edge element families for Maxwell's equations on hybrid quadrilateral/triangular meshes. Comput. Methods Appl. Mech. Engrg., 190:6709-6733, 2001.

[16] J. Fang. Time Domain Finite Difference Computation for Maxwell's Equations. PhD thesis, University of California at Berkeley, Berkeley, California, 1989.

[17] T. Deveze, L. Beaulie, and W. Tabbara. A fourth order scheme for the FDTD algorithm applied to Maxwell equtions. In Proceedings IEEE Antennas and Propagat. Soc. Int. Symp., pages 346-349, Chicago, IL, 1992. 
[18] M. F. Hadi and M. Piket-May. A modified FDTD $(2,4)$ scheme for modeling electrically large structures with high phase accuracy. IEEE Trans. Ant. Prop., 45(2):254-264, 1997.

[19] Z. Xie, C. Chan, and B. Zhang. An explicit fourth order orthogonal curvilinear staggered-grid FDTD method for Maxwell's equations. J. Comput. Phys., 175:739_ 763, 2002.

[20] R. L. Lee and N. K. Madsen. A mixed finite element formulation for Maxwell's equations in the time domain. J. Comput. Phys., 88:284-304, 1990.

[21] D. R. Lynch and K. D. Paulsen. Time domain integration of the maxwell equations on finite elements. IEEE Trans. Ant. Prop., 38:1933-1942, 1990.

[22] J. S. Hesthaven and T. Warburton. Nodal high-order methods on unstructured grids I. Time-domain solution of Maxwell's equations. J. Comput. Phys., 181(1):186-221, 2002.

[23] R. Graglia, D. Wilton, and A. Peterson. Higher order interpolatory vector bases for computational electromagnetics. IEEE Trans. Ant. Prop., 45(3):329-342, 1997.

[24] R. Rieben, D. White, and G. Rodrigue. Improved conditioning of finite element matrices using new high order interpolatory bases. IEEE Trans. Ant. Prop., August 2004. in press.

[25] J. C. Nédélec. Mixed finite elements in R3. Numer. Math., 35:315-341, 1980.

[26] R. Hiptmair. Canonical construction of finite elements. Math. Comp., 68(228):13251346, 1999.

[27] G. Rodrigue and D. White. A vector finite element time-domain method for solving maxwell's equations on unstructured hexahedral grids. SIAM J. Sci. Comp., 23(3):683-706, 2001.

[28] R. Rieben, D. White, and G. Rodrigue. High order symplectic integration methods for finite element solutions to time dependent maxwell equations. IEEE Trans. Ant. Prop., August 2004. in press.

[29] P. G. Ciarlet. The Finite Element Method for Elliptic Problems. North-Holland, 1978.

[30] M. Ainsworth and J. Coyle. Conditioning of hierarchic p-version Nedelec elements on meshes of curvilinear quadrilaterals and hexahedra. SIAM J. Num. Anal., 41(2):731$750,2003$.

[31] R. Rieben, D. White, and G. Rodrigue. Arbitrary order hierarchical vector bases for hexahedrons. In Proceedings of the 2003 IEEE International Antennas and Propagation Symposium, volume 2, pages 972-976, Columbus, Ohio, June 2003.

[32] P. R. Kotiuga. Helicity functionals and metric invariance in three dimensions. IEEE Trans. Mag., 25(4):2813-2815, 1989.

[33] J. A. Gallian. Contemporary Abstract Algebra. fourth edition, 1998.

[34] R. Richtmyer and K. Morton. Difference Methods for Initial Value Problems. John Wiley and Sons, New York, 1976.

[35] D. A. White and J. M. Koning. Computing solenoidal eigenmodes of the vector Helmholtz equation: a novel approach. IEEE Trans. Mag., 38(5):3420-3425, 2002. 
[36] D. Ruth. A canonical integration technique. IEEE Trans. Nuc. Sci., (4):2669-2671, 1983.

[37] J. Candy and W. Rozmus. A symplectic integration algorithm for seperable Hamiltonian functions. J. Comput. Phys., 92:230-256, 1991.

[38] C. Balanis. Advanced Engineering Electromagnetics. John Wiley and Sons, 1989. 